\title{
Existence Results for a Coupled System of Nonlinear Singular Fractional Differential Equations with Impulse Effects
}

\author{
Yuji Liu, ${ }^{1}$ Juan J. Nieto, ${ }^{2,3}$ and Óscar Otero-Zarraquiños ${ }^{2}$ \\ ${ }^{1}$ Department of Mathematics, Guangdong University of Business Studies, Guangzhou 510320, China \\ ${ }^{2}$ Departamento de Análisis Matemático, Facultad de Matemáticas, Universidad de Santiago de Compostela, \\ 15782 Santiago de Compostela, Spain \\ ${ }^{3}$ Department of Mathematics, Faculty of Science, King Abdulaziz University, P.O. Box 80203, Jeddah 21589, Saudi Arabia
}

Correspondence should be addressed to Juan J. Nieto; juanjose.nieto.roig@usc.es

Received 2 October 2012; Accepted 15 February 2013

Academic Editor: Jocelyn Sabatier

Copyright (C) 2013 Yuji Liu et al. This is an open access article distributed under the Creative Commons Attribution License, which permits unrestricted use, distribution, and reproduction in any medium, provided the original work is properly cited.

A boundary value problem for the singular fractional differential system with impulse effects is presented. By applying Schauder's fixed point theorem in a suitably Banach space, we obtain the existence of at least one solution for this problem. Two examples are presented to illustrate the main theorem.

\section{Introduction}

Fractional differential equations have received increasing attention during recent years since the behavior of many physical, chemical, and engineering processes can be properly described by using fractional differential equations theory; see the books [1-3], papers $[4,5]$ and references therein. For details on the geometric and physical interpretation of the derivatives of noninteger order, see, for example, [6-11]. For some recent works with applications to engineering we refer the reader to [12-15].

For an introduction of the basic theory of impulsive differential equation, we refer the reader to [16]. Among previous research, little is concerned with differential equations with fractional order with impulses [17]. Ahmad and Sivasundaram $[18,19]$ gave some existence results for twopoint boundary value problems involving nonlinear impulsive hybrid differential equations of fractional order $1<$ $\alpha \leq 2$. Ahmad and Nieto in [20] establish sufficient conditions for the existence of solutions of the antiperiodic boundary value problem for impulsive differential equations with the Caputo derivative of order $q \in(1,2]$. Some recent results on impulsive initial value problems or boundary value problems for fractional differential equations on a finite interval can be found in [21-23] and references therein. The memory property of fractional calculus makes studies more complicated.

This paper is motivated by [24] in which the following boundary value problem for the fractional differential equation

$$
\begin{array}{cc}
D_{0^{+}}^{\alpha} x(t)=f\left(t, y(t), D_{0^{+}}^{p} y(t)\right), & t \in(0,1), \\
D_{0^{+}}^{\beta} y(t)=g\left(t, x(t), D_{0^{+}}^{q} x(t)\right), & t \in(0,1), \\
x(0)=0, & y(0)=0, \quad x(1)-\gamma x(\eta)=0, \\
y(1)-\gamma y(\eta)=0
\end{array}
$$

was studied, where $1<\alpha, \beta<2,0<p \leq \beta-1$ and $0<$ $q \leq \alpha-1, \gamma>0,1>\gamma \eta^{\alpha-1}, 1>\gamma \eta^{\beta-1}$ and $f, g:[0,1] \times$ $R^{2} \rightarrow R$ are continuous functions, and $D_{0^{+}}$is the RiemannLiouville fractional derivative. An existence result was proved for BVP (1) in [24]. The growth assumptions imposed on $f$ and $g$ are sublinear cases (see [25, Theorem 3.1]); that is, there exist functions $a, b \in L^{1}(0,1)$, nonnegative constants $\epsilon_{1}, \epsilon_{2}>$ $0, \delta_{1}, \delta_{2} \geq 0$ and $\rho_{1}, \rho_{2}, \sigma_{1}, \sigma_{2} \in(0,1)$ such that

$$
\begin{aligned}
& |f(t, x, y)| \leq a(t)+\epsilon_{1}|x|^{\rho_{1}}+\epsilon_{2}|y|^{\rho_{2}}, \\
& |g(t, x, y)| \leq b(t)+\delta_{1}|x|^{\sigma_{1}}+\delta_{2}|y|^{\sigma_{2}} .
\end{aligned}
$$


In [25], the following boundary value problem for the fractional differential equation

$$
\begin{array}{ll}
D_{0^{+}}^{\alpha} x(t)=f\left(t, y(t), D_{0^{+}}^{p} y(t)\right), & t \in(0,1), \\
D_{0^{+}}^{\beta} y(t)=g\left(t, x(t), D_{0^{+}}^{q} x(t)\right), & t \in(0,1), \\
x(0)=0, \quad y(0)=0, \quad x(1)=0, & y(1)=0
\end{array}
$$

was studied, where $1<\alpha, \beta<2,0<p \leq \beta-1$ and $0<q \leq$ $\alpha-1$, and $f, g:[0,1] \times R^{2} \rightarrow R$ are continuous functions, and $D_{0^{+}}$is the Riemann-Liouville fractional derivative. The growth assumptions imposed on $f$ and $g$ are sublinear cases (see [25, Theorem 3.1]), that is, there exist functions $a, b \in$ $L^{1}(0,1)$, nonnegative constants $\epsilon_{1}, \epsilon_{2}>0, \delta_{1}, \delta_{2} \geq 0$, and $\rho_{1}, \rho_{2}, \sigma_{1}, \sigma_{2} \in(0,1]$ such that

$$
\begin{aligned}
& |f(t, x, y)| \leq a(t)+\epsilon_{1}|x|^{\rho_{1}}+\epsilon_{2}|y|^{\rho_{2}}, \\
& |g(t, x, y)| \leq b(t)+\delta_{1}|x|^{\sigma_{1}}+\delta_{2}|y|^{\sigma_{2}},
\end{aligned}
$$

or sublinear cases, that is, there exist nonnegative constants $\epsilon_{1}, \epsilon_{2}>0, \delta_{1}, \delta_{2} \geq 0$ and $\rho_{1}, \rho_{2}, \sigma_{1}, \sigma_{2} \in(1, \infty)$ such that

$$
\begin{aligned}
& |f(t, x, y)| \leq \epsilon_{1}|x|^{\rho_{1}}+\epsilon_{2}|y|^{\rho_{2}}, \\
& |g(t, x, y)| \leq \delta_{1}|x|^{\sigma_{1}}+\delta_{2}|y|^{\sigma_{2}} .
\end{aligned}
$$

We find that in the superlinear cases, BVP (3) has a pair of solutions $(x, y)=(0,0)$ without needing any other assumptions. Hence, these cases are trivial ones discussed in [25].

It is interesting to consider the solvability of BVP (1) when the growth assumptions imposed on $f, g$ are superlinear cases. Furthermore, the solvability of BVP (1) is not studied when $q>\alpha-1$ or $p>\beta-1$.

In this paper we consider the following nonlinear boundary value problem for the singular multiterm fractional differential equation with impulse effects whose boundary conditions are of integral form

$$
\begin{gathered}
D_{0^{+}}^{\alpha} x(t)=\phi(t) f\left(t, y(t), D_{0^{+}}^{p} y(t)\right), \\
t \in(0,1), \quad t \neq t_{1}, \\
D_{0^{+}}^{\beta} y(t)=\psi(t) g\left(t, x(t), D_{0^{+}}^{q} x(t)\right), \\
t \in(0,1), \quad t \neq t_{1}, \\
\lim _{t \rightarrow 0} t^{2-\alpha} x(t)=\int_{0}^{1} u(s) G\left(s, y(s), D_{0^{+}}^{p} y(s)\right) d s, \\
\lim _{t \rightarrow 0} t^{2-\beta} y(t)=\int_{0}^{1} v(s) H\left(s, x(s), D_{0^{+}}^{q} x(s)\right) d s, \\
x(1)=\int_{0}^{1} m(s) M\left(s, y(s), D_{0^{+}}^{p} y(s)\right) d s,
\end{gathered}
$$

$$
\begin{gathered}
y(1)=\int_{0}^{1} n(s) N\left(s, x(s), D_{0^{+}}^{q} x(s)\right) d s \\
\Delta x\left(t_{1}\right)=\lim _{t \rightarrow t_{1}^{+}} x(t)-\lim _{t \rightarrow t_{1}^{-}} x(t)=I\left(t_{1}, y\left(t_{1}\right), D_{0^{+}}^{p} y\left(t_{1}\right)\right), \\
\Delta y\left(t_{1}\right)=\lim _{t \rightarrow t_{1}^{+}} y(t)-\lim _{t \rightarrow t_{1}^{-}} y(t)=J\left(t_{1}, x\left(t_{1}\right), D_{0^{+}}^{q} x\left(t_{1}\right)\right), \\
\Delta D_{0^{+}}^{q} x\left(t_{1}\right)=\lim _{t \rightarrow t_{1}^{+}} D_{0^{+}}^{q} x(t)-\lim _{t \rightarrow t_{1}^{-}} D_{0^{+}}^{q} x(t) \\
=I_{1}\left(t_{1}, y\left(t_{1}\right), D_{0^{+}}^{p} y\left(t_{1}\right)\right) \\
\Delta D_{0^{+}}^{p} y\left(t_{1}\right)=\lim _{t \rightarrow t_{1}^{+}} D_{0^{+}}^{p} y(t)-\lim _{t \rightarrow t_{1}^{-}} D_{0^{+}}^{p} y(t) \\
=J_{1}\left(t_{1}, x\left(t_{1}\right), D_{0^{+}}^{q} x\left(t_{1}\right)\right),
\end{gathered}
$$

where

(a) $1<\alpha, \beta \leq 2,0<p<\beta$ and $0<q<\alpha, D_{0^{+}}$is the Riemann-Liouville fractional derivative,

(b) $\phi, \psi:(0,1) \rightarrow R, f, g$ defined on $(0,1) \times R^{2}$,

(c) $m, n, u, v:(0,1) \rightarrow R$ with $m, n, u, v \in L^{1}(0,1)$, $G, H, M, N$ defined on $(0,1) \times R^{2}$,

(d) $0=t_{0}<t_{1}<t_{2}=1$,

(e) $I, I_{1}, J, J_{1}:(0,1) \times R^{2} \rightarrow R$.

A pair of functions $(x, y)$ defined on $(0,1)$ is called a solution of BVP (1) and BVP (3), if $\left.x\right|_{\left(t_{k}, t_{k+1}\right]},\left.D_{0^{+}}^{q} x\right|_{\left(t_{k}, t_{k+1}\right]}$ and $\left.y\right|_{\left(t_{k}, t_{k+1}\right]},\left.D_{0^{+}}^{p} y\right|_{\left(t_{k}, t_{k+1}\right]}(k=0,1)$ are continuous, there exists the limits

$$
\begin{array}{r}
\lim _{t \rightarrow t_{k}^{+}} t^{2-\alpha} x(t), \quad \lim _{t \rightarrow t_{k}^{+}} t^{2-\beta} y(t), \\
\lim _{t \rightarrow t_{k}^{+}} t^{2+q-\alpha} D_{0^{+}}^{q} x(t), \quad \lim _{t \rightarrow t_{k}^{+}} t^{2+p-\beta} D_{0^{+}}^{p} y(t), \\
k=0,1,
\end{array}
$$

$D_{0^{+}}^{\alpha} x, D_{0^{+}}^{\beta} y \in L^{1}(0,1)$ and $(x, y)$ satisfies all equations in (6) and (7).

The novelty of this paper is as follows: first, the fractional differential equations in (6) are multiterm ones and their nonlinearities $f, g$ depend on the lower fractional derivatives; second, both $\phi$ and $\psi$ may be singular at $t=0$ and $t=1$, that is, $\phi(t) f(t, x, y)$ and $\psi(t) g(t, x, y)$ may be not continuous functions on $[0,1] \times R^{2}$, the boundary conditions are integral boundary conditions, and we obtain the results on the existence of at least one solution of BVP (6)-(7); third, $0<p<\beta$ and $0<q<\alpha$ are supposed; the growth assumptions imposed on $f, g, G, H, M, N$ and $I, I_{1}, J, J_{1}$ are allowed to be sublinear cases. Finally, two examples are given to illustrate the efficiency of the main theorem.

The remainder of this paper is as follows: in Section 2, we present preliminary results. In Section 3, the main theorem and its proof are given. In Section 4, two examples are given to illustrate the main results. 


\section{Preliminaries}

In this section, we present some background definitions and preliminary results.

Definition 1 (see [1]). The Riemann-Liouville fractional integral of order $\alpha>0$ of a function $g:(0, \infty) \rightarrow R$ is given by

$$
I_{0^{+}}^{\alpha} g(t)=\frac{1}{\Gamma(\alpha)} \int_{0}^{t}(t-s)^{\alpha-1} g(s) d s,
$$

provided that the right-hand side exists.

Definition 2 (see [1]). The Riemann-Liouville fractional derivative of order $\alpha>0$ of a continuous function $g$ : $(0, \infty) \rightarrow R$ is given by

$$
D_{0^{+}}^{\alpha} g(t)=\frac{1}{\Gamma(n-\alpha)} \frac{d^{n}}{d t^{n}} \int_{0}^{t} \frac{g(s)}{(t-s)^{\alpha-n+1}} d s,
$$

where $n-1 \leq \alpha<n$, provided that the right-hand side is pointwise defined on $(0, \infty)$.

Definition 3. $K:(0,1) \times R^{2} \rightarrow R$ is called a $\beta$-Caratheodory function if $K$ satisfies that

(i) $t \rightarrow K\left(t, t^{\beta-2} U, t^{\beta-p-2} V\right)$ is continuous on $\left(t_{k}, t_{k+1}\right](k=0,1)$ for every $(U, V) \in R^{2}$;

(ii) $(U, V) \rightarrow K\left(t, t^{\beta-2} U, t^{\beta-p-2} V\right)$ is continuous on $R^{2}$ for every $t \in(0,1)$;

(iii) for each $r>0$ there exists a constant $A_{r}>0$ such that $\left|K\left(t, t^{\beta-2} U, t^{\beta-p-2} V\right)\right| \leq A_{r}, t \in(0,1),|U|,|V| \leq r$.

Definition 4. $Q:(0,1) \times R^{2} \rightarrow R$ is called a $\alpha$-Caratheodory function if $Q$ satisfies that

(i) $t \quad \rightarrow \quad Q\left(t, t^{\alpha-2} U, t^{\alpha-q-2} V\right)$ is continuous on $\left(t_{k}, t_{k+1}\right](k=0,1)$ for every $(U, V) \in R^{2}$;

(ii) $(U, V) \rightarrow Q\left(t, t^{\alpha-2} U, t^{\alpha-q-2} V\right)$ is continuous on $R^{2}$ for every $t \in(0,1)$;

(iii) for each $r>0$ there exists a constant $B_{r}>0$ such that $\left|Q\left(t, t^{\alpha-2} U, t^{\alpha-q-2} V\right)\right| \leq B_{r}, t \in(0,1),|U|,|V| \leq r$.

Lemma 5 (the Leray-Schauder nonlinear alternative [23]). Let $X$ be a Banach space and $T: X \rightarrow X$ be a completely continuous operator. Suppose $\Omega$ is a nonempty open subset of $X$ centered at zero. Then either there exists $x \in \partial \Omega$ and $\lambda \in(0,1)$ such that $x=\lambda T x$ or there exists $x \in \bar{\Omega}$ such that $x=T x$.

Let the gamma and beta functions $\Gamma(\alpha)$ and $\mathbf{B}(p, q)$ be defined by

$$
\begin{gathered}
\Gamma(\alpha)=\int_{0}^{+\infty} x^{\alpha-1} e^{-x} d x, \\
\mathbf{B}(p, q)=\int_{0}^{1} x^{p-1}(1-x)^{q-1} d x, \\
\|m\|_{1}=\int_{0}^{1}|m(s)| d s \quad \text { for } m \in L^{1}(0,1) .
\end{gathered}
$$

\section{Choose}

X

$$
=\left\{\begin{array}{c}
\left.x\right|_{\left(t_{k}, t_{k+1}\right]} \in C^{0}\left(t_{k}, t_{k+1}\right](k=0,1), \\
\left.D_{0^{+}}^{q} x\right|_{\left(t_{k}, t_{k+1}\right]} \in C^{0}\left(t_{k}, t_{k+1}\right](k=0,1), \\
\text { there exist the limits } \\
\lim _{t \rightarrow t_{k}^{+}} t^{2-\alpha} x(t), \\
\lim _{t \rightarrow t_{k}^{+}} t^{2+q-\alpha} D_{0^{+}}^{q} x(t)
\end{array}\right\},
$$

Y

$$
=\left\{\begin{array}{c}
\left.y\right|_{\left(t_{k}, t_{k+1}\right]} \in C^{0}\left(t_{k}, t_{k+1}\right](k=0,1), \\
\left.D_{0^{+}}^{p} x\right|_{\left(t_{k}, t_{k+1}\right]} \in C^{0}\left(t_{k}, t_{k+1}\right](k=0,1), \\
y:(0,1] \longrightarrow R \quad \text { there exist the limits } \\
\lim _{t \rightarrow t_{k}^{+}} t^{2-\beta} y(t), \\
\lim _{t \rightarrow t_{k}^{+}} t^{2+p-\beta} D_{0^{+}}^{p} y(t)
\end{array}\right\} .
$$

For $x \in X$, define the norm by

$$
\begin{aligned}
\|x\| & =\|x\|_{X} \\
& =\max \left\{\sup _{t \in(0,1)} t^{2-\alpha}|x(t)|, \sup _{t \in(0,1)} t^{2+q-\alpha}\left|D_{0^{+}}^{q} x(t)\right|\right\} .
\end{aligned}
$$

It is easy to show that $X$ is a real Banach space. For $y \in Y$, define the norm by

$$
\begin{aligned}
\|y\| & =\|y\|_{Y} \\
& =\max \left\{\sup _{t \in(0,1)} t^{2-\beta}|y(t)|, \sup _{t \in(0,1)} t^{2+p-\beta}\left|D_{0^{+}}^{p} y(t)\right|\right\} .
\end{aligned}
$$

It is easy to show that $Y$ is a real Banach space. Thus, $(X \times$ $Y,\|\cdot\|)$ is a Banach space with the norm defined by $\|(x, y)\|=$ $\max \left\{\|x\|_{X},\|y\|_{Y}\right\}$ for $(x, y) \in X \times Y$.

In this paper, we suppose the following:

(A) $\phi$ satisfies that there exist constants $L_{1}>0, k>-1$, $\delta \in(q-\alpha, 0]$ such that $\alpha+2 \delta-q>0, \alpha+k+\delta-q \geq 0$, and $|\phi(t)| \leq L_{1} t^{k}(1-t)^{\delta}$ for all $t \in(0,1) ; \psi$ satisfies that there exist constants $L_{2}>0, l>-1, \theta \in(p-\beta, 0]$ such that $\beta+2 \theta-p>0, \beta+l+\theta-p \geq 0$, and $|\psi(t)| \leq L_{2} t^{l}(1-t)^{\theta}$ for all $t \in(0,1)$.

(B) $f, G, M, I, I_{1}$ are $\beta$-Caratheodory functions and $g, H$, $N, J, J_{1}$ are $\alpha$-Caratheodory functions.

Remark 6. Suppose that $f$ is a $\beta$-Caratheodory function. For example, $\alpha=7 / 4, q=1 / 8$, choose $k=-1 / 2, \delta=-3 / 4$ and $\phi(t)=t^{k}(1-t)^{\delta}$, then $k>-1, \delta \in(-\alpha, 0]$ such that $\alpha+2 \delta-q>0, \alpha+k+\delta-q \geq 0$, and $|\phi(t)| \leq t^{k}(1-t)^{\delta}$ for all $t \in(0,1)$. It is easy to see that $\phi$ is singular at $t=0$ and $t=1$. 
Lemma 7. Suppose that $y \in Y$, and (a)-(e), (A)-(B) hold. Then $x \in X$ is a solution of

$$
\begin{gathered}
D_{0^{+}}^{\alpha} x(t)=\phi(t) f\left(t, y(t), D_{0^{+}}^{p} y(t)\right), \quad t \in(0,1), t \neq t_{1}, \\
\lim _{t \rightarrow 0} t^{2-\alpha} x(t)=\int_{0}^{1} u(s) G\left(s, y(s), D_{0^{+}}^{p} y(s)\right) d s \\
x(1)=\int_{0}^{1} m(s) M\left(s, y(s), D_{0^{+}}^{p} y(s)\right) d s, \\
\Delta x\left(t_{1}\right)=I\left(t_{1}, y\left(t_{1}\right), D_{0^{+}}^{p} y\left(t_{1}\right)\right), \\
\Delta D_{0^{+}}^{q} x\left(t_{1}\right)=I_{1}\left(t_{1}, y\left(t_{1}\right), D_{0^{+}}^{p} y\left(t_{1}\right)\right),
\end{gathered}
$$

if and only if $x \in X$ satisfies the integral equation

$$
\begin{cases}\int_{0}^{t} & \frac{(t-s)^{\alpha-1}}{\Gamma(\alpha)} \phi(u) f\left(u, y(u), D_{0^{+}}^{p} y(u)\right) d u \\ & -\frac{t^{\alpha-1}}{\Gamma(\alpha)} \\ & \times \int_{0}^{1}(1-s)^{\alpha-1} \phi(s) f\left(s, y(s), D_{0^{+}}^{p} y(s)\right) d s \\ & +t^{\alpha-2} \int_{0}^{1} u(s) G\left(s, y(s), D_{0^{+}}^{p} y(s)\right) d s \\ & +t^{\alpha-1} \int_{0}^{1} m(s) M\left(s, y(s), D_{0^{+}}^{p} y(s)\right) d s \\ & +\frac{t^{\alpha-1}}{\Pi} \\ & \times\left(\frac{\Gamma(\alpha)}{\Gamma(\alpha-q)} t_{1}^{\alpha-q-1}-\frac{\Gamma(\alpha-1)}{\Gamma(\alpha-q-2)} t_{1}^{\alpha-q-2}\right) \\ & \times I\left(t_{1}, y\left(t_{1}\right), D_{0^{+}}^{p} y\left(t_{1}\right)\right) \\ & +\frac{t^{\alpha-2}-t^{\alpha-1}}{\Pi} t_{1}^{\alpha-1} I_{1}\left(t_{1}, y\left(t_{1}\right), D_{0^{+}}^{p} y\left(t_{1}\right)\right), \\ & +\frac{t^{\alpha-1}\left(t_{1}^{\alpha-2}-t_{1}^{\alpha-1}\right)}{\Pi} \\ & \times I_{1}\left(t_{1}, y\left(t_{1}\right), D_{0^{+}}^{p} y\left(t_{1}\right)\right), \quad t \in\left(0, t_{1}\right], \\ & +t^{\alpha-1} \int_{0}^{1} m(s) M\left(s, y(s), D_{0^{+}}^{p} y(s)\right) d s \\ & \frac{(t-s)^{\alpha-1}}{\Gamma(\alpha)} \phi(s) f\left(s, y(s), D_{0^{+}}^{p} y(s)\right) d s \\ & +\frac{t^{\alpha-1}-t^{\alpha-2}}{\Gamma(\alpha)} \frac{\Gamma(\alpha)}{\Gamma(\alpha-q)} t_{1}^{\alpha-q-1} \\ & +\left(t_{0}^{1}(1-s)^{\alpha-1} \phi(s) f\left(s, y(s), D_{0^{+}}^{p} y(s)\right) d s\right.\end{cases}
$$

where

$$
\Pi=\left(\frac{\Gamma(\alpha-1)}{\Gamma(\alpha-q-1)}-\frac{\Gamma(\alpha)}{\Gamma(\alpha-q)}\right) t_{1}^{2 \alpha-q-3} .
$$

Proof. If $y \in Y$ is a solution of BVP (15), then

$$
\begin{aligned}
\|y\| & =\max \left\{\sup _{t \in(0,1)} t^{2-\beta}|y(t)|, \sup _{t \in(0,1)} t^{2+p-\beta}\left|D_{0^{+}}^{p} y(t)\right|\right\} \\
& =r<+\infty
\end{aligned}
$$

and $x$ satisfies all equations in (31) From (B), $f$ is a $\beta$ Caratheodory function, then there exists $A_{r}>0$ such that

$$
\begin{aligned}
& \left|f\left(t, y(t), D_{0^{+}}^{p} y(t)\right)\right| \\
& \quad=\left|f\left(t, t^{\beta-2} t^{2-\beta} y(t), t^{\beta-p-2} t^{2+p-\beta} D_{0^{+}}^{p} y(t)\right)\right| \leq A_{r} .
\end{aligned}
$$

Similarly we get that there exist constants $A_{r}^{\prime}, A_{r}^{\prime \prime}, B_{r}^{\prime}, B_{r}^{\prime \prime}>0$ such that

$$
\begin{gathered}
\left|G\left(t, y(t), D_{0^{+}}^{p} y(t)\right)\right| \leq A_{r}^{\prime}, \\
\left|M\left(t, y(t), D_{0^{+}}^{p} y(t)\right)\right| \leq A_{r}^{\prime \prime}, \\
t \in(0,1), \\
\left|I\left(t_{1}, y\left(t_{1}\right), D_{0^{+}}^{p} y\left(t_{1}\right)\right)\right| \leq B_{r}^{\prime}, \\
\left|I_{1}\left(t_{1}, y\left(t_{1}\right), D_{0^{+}}^{p} y\left(t_{1}\right)\right)\right| \leq B_{r}^{\prime \prime} .
\end{gathered}
$$

It follows from (15) that, for $t \in\left(t_{k}, t_{k+1}\right](k=0,1)$, there exist constants $c_{k}, d_{k} \in R$ such that

$$
\begin{aligned}
x(t)= & \frac{1}{\Gamma(\alpha)} \int_{0}^{t}(t-s)^{\alpha-1} \phi(s) f\left(s, y(s), D_{0^{+}}^{p} y(s)\right) d s \\
& +c_{k} t^{\alpha-1}+d_{k} t^{\alpha-2}, \quad t \in\left(t_{k}, t_{k+1}\right], k=0,1 .
\end{aligned}
$$
get

From $\lim _{t \rightarrow 0} t^{2-\alpha} x(t)=\int_{0}^{1} u(s) G\left(s, y(s), D_{0^{+}}^{p} y(s)\right) d s$, we

$$
d_{0}=\int_{0}^{1} u(s) G\left(s, y(s), D_{0^{+}}^{p} y(s)\right) d s
$$

From $x(1)=\int_{0}^{1} m(s) M\left(s, y(s), D_{0^{+}}^{p} y(s)\right) d s$, we get

$$
\begin{aligned}
& \frac{1}{\Gamma(\alpha)} \int_{0}^{1}(1-s)^{\alpha-1} \phi(s) f\left(s, y(s), D_{0^{+}}^{p} y(s)\right) d s+c_{1}+d_{1} \\
& \quad=\int_{0}^{1} m(s) M\left(s, y(s), D_{0^{+}}^{p} y(s)\right) d s .
\end{aligned}
$$

From $\Delta x\left(t_{1}\right)=I\left(t_{1}, y\left(t_{1}\right), D_{0^{+}}^{p} y\left(t_{1}\right)\right)$, we get

$\left(c_{1}-c_{0}\right) t_{1}^{\alpha-1}+\left(d_{1}-d_{0}\right) t_{1}^{\alpha-2}=I\left(t_{1}, y\left(t_{1}\right), D_{0^{+}}^{p} y\left(t_{1}\right)\right)$. 
From $\Delta D_{0^{+}}^{q} x\left(t_{1}\right)=I_{1}\left(t_{1}, y\left(t_{1}\right), D_{0^{+}}^{p} y\left(t_{1}\right)\right)$, we get

$$
\begin{aligned}
& \left(c_{1}-c_{0}\right) \frac{\Gamma(\alpha)}{\Gamma(\alpha-q)} t_{1}^{\alpha-q-1}+\left(d_{1}-d_{0}\right) \frac{\Gamma(\alpha-1)}{\Gamma(\alpha-q-2)} t_{1}^{\alpha-q-2} \\
& =I_{1}\left(t_{1}, y\left(t_{1}\right), D_{0^{+}}^{p} y\left(t_{1}\right)\right) .
\end{aligned}
$$

It follows that

$$
\begin{aligned}
c_{1}-c_{0}= & \left(\frac{\Gamma(\alpha-1)}{\Gamma(\alpha-q-2)} t_{1}^{\alpha-q-2} I\left(t_{1}, y\left(t_{1}\right), D_{0^{+}}^{p} y\left(t_{1}\right)\right)\right. \\
& \left.-t_{1}^{\alpha-2} I_{1}\left(t_{1}, y\left(t_{1}\right), D_{0^{+}}^{p} y\left(t_{1}\right)\right)\right) \times(\Pi)^{-1}, \\
d_{1}-d_{0}= & \left(t_{1}^{\alpha-1} I_{1}\left(t_{1}, y\left(t_{1}\right), D_{0^{+}}^{p} y\left(t_{1}\right)\right)\right. \\
& \left.-\frac{\Gamma(\alpha)}{\Gamma(\alpha-q)} t_{1}^{\alpha-q-1} I\left(t_{1}, y\left(t_{1}\right), D_{0^{+}}^{p} y\left(t_{1}\right)\right)\right) \\
& \times(\Pi)^{-1} .
\end{aligned}
$$

Then

$$
\begin{aligned}
d_{1}= & \left(t_{1}^{\alpha-1} I_{1}\left(t_{1}, y\left(t_{1}\right), D_{0^{+}}^{p} y\left(t_{1}\right)\right)\right. \\
& \left.-\frac{\Gamma(\alpha)}{\Gamma(\alpha-q)} t_{1}^{\alpha-q-1} I\left(t_{1}, y\left(t_{1}\right), D_{0^{+}}^{p} y\left(t_{1}\right)\right)\right) \times(\Pi)^{-1} \\
& +\int_{0}^{1} u(s) G\left(s, y(s), D_{0^{+}}^{p} y(s)\right) d s .
\end{aligned}
$$

So

$$
\begin{aligned}
c_{1}= & \int_{0}^{1} m(s) M\left(s, y(s), D_{0^{+}}^{p} y(s)\right) d s \\
& -\frac{1}{\Gamma(\alpha)} \int_{0}^{1}(1-s)^{\alpha-1} \phi(s) f\left(s, y(s), D_{0^{+}}^{p} y(s)\right) d s \\
& -\left(t_{1}^{\alpha-1} I_{1}\left(t_{1}, y\left(t_{1}\right), D_{0^{+}}^{p} y\left(t_{1}\right)\right)\right. \\
& \left.-\frac{\Gamma(\alpha)}{\Gamma(\alpha-q)} t_{1}^{\alpha-q-1} I\left(t_{1}, y\left(t_{1}\right), D_{0^{+}}^{p} y\left(t_{1}\right)\right)\right) \times(\Pi)^{-1} \\
c_{0}= & \int_{0}^{1} m(s) M\left(s, y(s), D_{0^{+}}^{p} y(s)\right) d s \\
& -\frac{1}{\Gamma(\alpha)} \int_{0}^{1}(1-s)^{\alpha-1} \phi(s) f\left(s, y(s), D_{0^{+}}^{p} y(s)\right) d s \\
& -\left(t_{1}^{\alpha-1} I_{1}\left(t_{1}, y\left(t_{1}\right), D_{0^{+}}^{p} y\left(t_{1}\right)\right)\right. \\
& \left.\quad-\frac{\Gamma(\alpha)}{\Gamma(\alpha-q)} t_{1}^{\alpha-q-1} I\left(t_{1}, y\left(t_{1}\right), D_{0^{+}}^{p} y\left(t_{1}\right)\right)\right) \times(\Pi)^{-1}
\end{aligned}
$$

$$
\begin{aligned}
& -\int_{0}^{1} u(s) G\left(s, y(s), D_{0^{+}}^{p} y(s)\right) d s \\
& -\left(\frac{\Gamma(\alpha-1)}{\Gamma(\alpha-q-2)} t_{1}^{\alpha-q-2} I\left(t_{1}, y\left(t_{1}\right), D_{0^{+}}^{p} y\left(t_{1}\right)\right)\right. \\
& \left.\quad-t_{1}^{\alpha-2} I_{1}\left(t_{1}, y\left(t_{1}\right), D_{0^{+}}^{p} y\left(t_{1}\right)\right)\right) \times(\Pi)^{-1} .
\end{aligned}
$$

Hence, for $t \in\left(0, t_{1}\right]$, we have

$$
\begin{aligned}
x(t)= & \int_{0}^{t} \frac{(t-s)^{\alpha-1}}{\Gamma(\alpha)} \phi(u) f\left(u, y(u), D_{0^{+}}^{p} y(u)\right) d u \\
& -\frac{t^{\alpha-1}}{\Gamma(\alpha)} \int_{0}^{1}(1-s)^{\alpha-1} \phi(s) f\left(s, y(s), D_{0^{+}}^{p} y(s)\right) d s \\
& +t^{\alpha-2} \int_{0}^{1} u(s) G\left(s, y(s), D_{0^{+}}^{p} y(s)\right) d s \\
& +t^{\alpha-1} \int_{0}^{1} m(s) M\left(s, y(s), D_{0^{+}}^{p} y(s)\right) d s \\
& +\frac{t^{\alpha-1}}{\Pi}\left(\frac{\Gamma(\alpha)}{\Gamma(\alpha-q)} t_{1}^{\alpha-q-1}-\frac{\Gamma(\alpha-1)}{\Gamma(\alpha-q-2)} t_{1}^{\alpha-q-2}\right) \\
& \times I\left(t_{1}, y\left(t_{1}\right), D_{0^{+}}^{p} y\left(t_{1}\right)\right) \\
& +\frac{t^{\alpha-1}\left(t_{1}^{\alpha-2}-t_{1}^{\alpha-1}\right)}{\Pi} I_{1}\left(t_{1}, y\left(t_{1}\right), D_{0^{+}}^{p} y\left(t_{1}\right)\right) .
\end{aligned}
$$

And for $t \in\left(t_{1}, 1\right]$, we have

$$
\begin{aligned}
x(t)= & \int_{0}^{t} \frac{(t-s)^{\alpha-1}}{\Gamma(\alpha)} \phi(s) f\left(s, y(s), D_{0^{+}}^{p} y(s)\right) d s \\
& -\frac{t^{\alpha-1}}{\Gamma(\alpha)} \int_{0}^{1}(1-s)^{\alpha-1} \phi(s) f\left(s, y(s), D_{0^{+}}^{p} y(s)\right) d s \\
& +\left(t^{\alpha-2}-t^{\alpha-1}\right) \int_{0}^{1} u(s) G\left(s, y(s), D_{0^{+}}^{p} y(s)\right) d s \\
& +t^{\alpha-1} \int_{0}^{1} m(s) M\left(s, y(s), D_{0^{+}}^{p} y(s)\right) d s \\
& +\frac{t^{\alpha-1}-t^{\alpha-2}}{\Pi} \frac{\Gamma(\alpha)}{\Gamma(\alpha-q)} t_{1}^{\alpha-q-1} \\
& \times I\left(t_{1}, y\left(t_{1}\right), D_{0^{+}}^{p} y\left(t_{1}\right)\right) \\
& +\frac{t^{\alpha-2}-t^{\alpha-1}}{\Pi} t_{1}^{\alpha-1} I_{1}\left(t_{1}, y\left(t_{1}\right), D_{0^{+}}^{p} y\left(t_{1}\right)\right) .
\end{aligned}
$$

Hence, $x \in X$ satisfies (16).

On the other hand, if $y \in Y$ and $x \in X$ is a solution of (16), then we can prove that $x \in X$ is a solution of BVP (6)-(7). The proof is completed. 
Lemma 8. Suppose that $x \in X$, and (a)-(e), (A)-(B) hold. Then $y \in Y$ is a solution of

$$
\begin{gathered}
D_{0^{+}}^{\beta} y(t)=\psi(t) g\left(t, x(t), D_{0^{+}}^{q} x(t)\right), \quad t \in(0,1), t \neq t_{1}, \\
\lim _{t \rightarrow 0} t^{2-\beta} y(t)=\int_{0}^{1} v(s) H\left(s, x(s), D_{0^{+}}^{q} x(s)\right) d s, \\
y(1)=\int_{0}^{1} n(s) N\left(s, x(s), D_{0^{+}}^{q} x(s)\right) d s, \\
\Delta y\left(t_{1}\right)=J\left(t_{1}, x\left(t_{1}\right), D_{0^{+}}^{q} x\left(t_{1}\right)\right), \\
\Delta D_{0^{+}}^{p} y\left(t_{1}\right)=J_{1}\left(t_{1}, x\left(t_{1}\right), D_{0^{+}}^{q} x\left(t_{1}\right)\right),
\end{gathered}
$$

if and only if $y \in Y$ satisfies the integral equation

$$
\begin{aligned}
& \iint_{0}^{t} \frac{(t-s)^{\beta-1}}{\Gamma(\beta)} \psi(u) g\left(u, x(u), D_{0^{+}}^{q} x(u)\right) d u \\
& -\frac{t^{\beta-1}}{\Gamma(\beta)} \\
& \times \int_{0}^{1}(1-s)^{\beta-1} \psi(s) g\left(s, x(s), D_{0^{+}}^{q} x(s)\right) d s \\
& +t^{\beta-2} \\
& \times \int_{0}^{1} v(s) H\left(s, y(s), D_{0^{+}}^{p} y(s)\right) d s \\
& +t^{\beta-1} \\
& \times \int_{0}^{1} n(s) N\left(s, x(s), D_{0^{+}}^{q} x(s)\right) d s \\
& +\frac{t^{\beta-1}}{\Xi} \\
& \times\left(\frac{\Gamma(\beta)}{\Gamma(\beta-p)} t_{1}^{\beta-p-1}-\frac{\Gamma(\beta-1)}{\Gamma(\beta-p-2)} t_{1}^{\beta-p-2}\right) \\
& \times J\left(t_{1}, x\left(t_{1}\right), D_{0^{+}}^{q} x\left(t_{1}\right)\right) \\
& +\frac{t^{\beta-1}\left(t_{1}^{\beta-2}-t_{1}^{\beta-1}\right)}{\Xi} \\
& y(t)=\left\{\quad \times J_{1}\left(t_{1}, x\left(t_{1}\right), D_{0^{+}}^{q} x\left(t_{1}\right)\right), \quad t \in\left(0, t_{1}\right],\right. \\
& \int_{0}^{t} \frac{(t-s)^{\beta-1}}{\Gamma(\beta)} \psi(s) g\left(s, x(s), D_{0^{+}}^{q} x(s)\right) d s \\
& -\frac{t^{\beta-1}}{\Gamma(\beta)} \\
& \times \int_{0}^{1}(1-s)^{\beta-1} \psi(s) g\left(s, x(s), D_{0^{+}}^{q} x(s)\right) d s \\
& +\left(t^{\beta-2}-t^{\beta-1}\right) \\
& \times \int_{0}^{1} v(s) H\left(s, y(s), D_{0^{+}}^{p} y(s)\right) d s \\
& +t^{\beta-1} \\
& \times \int_{0}^{1} n(s) N\left(s, x(s), D_{0^{+}}^{q} x(s)\right) d s \\
& +\frac{t^{\beta-1}-t^{\beta-2}}{\Xi} \frac{\Gamma(\beta)}{\Gamma(\beta-p)} t_{1}^{\beta-p-1} \\
& \times J\left(t_{1}, x\left(t_{1}\right), D_{0^{+}}^{q} x\left(t_{1}\right)\right) \\
& +\frac{t^{\beta-2}-t^{\beta-1}}{\Xi} t_{1}^{\beta-1} \\
& \times J_{1}\left(t_{1}, x\left(t_{1}\right), D_{0^{+}}^{q} x\left(t_{1}\right)\right), \quad t \in\left(t_{1}, 1\right],
\end{aligned}
$$

where

$$
\Xi=\left(\frac{\Gamma(\beta-1)}{\Gamma(\beta-p-1)}-\frac{\Gamma(\beta)}{\Gamma(\beta-p)}\right) t_{1}^{2 \beta-p-3} .
$$

Proof. The proof is similar to that of the proof of Lemma 7 and is omitted.

Now, we define the operator $T$ on $X \times Y$ by $T(x, y)(t)=$ $\left(\left(T_{1} y\right)(t),\left(T_{2} x\right)(t)\right)$ with

$\left(T_{1} y\right)(t)$

$$
\begin{aligned}
& \iint_{0}^{t} \frac{(t-s)^{\alpha-1}}{\Gamma(\alpha)} \phi(u) f\left(u, y(u), D_{0^{+}}^{p} y(u)\right) d u \\
& -\frac{t^{\alpha-1}}{\Gamma(\alpha)} \int_{0}^{1}(1-s)^{\alpha-1} \phi(s) f\left(s, y(s), D_{0^{+}}^{p} y(s)\right) d s \\
& +t^{\alpha-2} \int_{0}^{1} u(s) G\left(s, y(s), D_{0^{+}}^{p} y(s)\right) d s \\
& +t^{\alpha-1} \int_{0}^{1} m(s) M\left(s, y(s), D_{0^{+}}^{p} y(s)\right) d s \\
& +\frac{t^{\alpha-1}}{\Pi}\left(\frac{\Gamma(\alpha)}{\Gamma(\alpha-q)} t_{1}^{\alpha-q-1}-\frac{\Gamma(\alpha-1)}{\Gamma(\alpha-q-2)} t_{1}^{\alpha-q-2}\right) \\
& \times I\left(t_{1}, y\left(t_{1}\right), D_{0^{+}}^{p} y\left(t_{1}\right)\right) \\
& +\frac{t^{\alpha-1}\left(t_{1}^{\alpha-2}-t_{1}^{\alpha-1}\right)}{\Pi} \\
& \times I_{1}\left(t_{1}, y\left(t_{1}\right), D_{0^{+}}^{p} y\left(t_{1}\right)\right), \quad t \in\left(0, t_{1}\right], \\
& \int_{0}^{t} \frac{(t-s)^{\alpha-1}}{\Gamma(\alpha)} \phi(s) f\left(s, y(s), D_{0^{+}}^{p} y(s)\right) d s \\
& -\frac{t^{\alpha-1}}{\Gamma(\alpha)} \int_{0}^{1}(1-s)^{\alpha-1} \phi(s) f\left(s, y(s), D_{0^{+}}^{p} y(s)\right) d s \\
& +\left(t^{\alpha-2}-t^{\alpha-1}\right) \int_{0}^{1} u(s) G\left(s, y(s), D_{0^{+}}^{p} y(s)\right) d s \\
& +t^{\alpha-1} \int_{0}^{1} m(s) M\left(s, y(s), D_{0^{+}}^{p} y(s)\right) d s \\
& +\frac{t^{\alpha-1}-t^{\alpha-2}}{\Pi} \frac{\Gamma(\alpha)}{\Gamma(\alpha-q)} t_{1}^{\alpha-q-1} I\left(t_{1}, y\left(t_{1}\right), D_{0^{+}}^{p} y\left(t_{1}\right)\right) \\
& +\frac{t^{\alpha-2}-t^{\alpha-1}}{\Pi} t_{1}^{\alpha-1} I_{1}\left(t_{1}, y\left(t_{1}\right), D_{0^{+}}^{p} y\left(t_{1}\right)\right), \\
& t \in\left(t_{1}, 1\right],
\end{aligned}
$$




$$
\begin{aligned}
& \left(T_{2} x\right)(t) \\
& \int_{0}^{t} \frac{(t-s)^{\beta-1}}{\Gamma(\beta)} \psi(u) g\left(u, x(u), D_{0^{+}}^{q} x(u)\right) d u \\
& -\frac{t^{\beta-1}}{\Gamma(\beta)} \int_{0}^{1}(1-s)^{\beta-1} \psi(s) g\left(s, x(s), D_{0^{+}}^{q} x(s)\right) d s \\
& +t^{\beta-2} \int_{0}^{1} v(s) H\left(s, y(s), D_{0^{+}}^{p} y(s)\right) d s \\
& +t^{\beta-1} \int_{0}^{1} n(s) N\left(s, x(s), D_{0^{+}}^{q} x(s)\right) d s \\
& +\frac{t^{\beta-1}}{\Xi}\left(\frac{\Gamma(\beta)}{\Gamma(\beta-p)} t_{1}^{\beta-p-1}-\frac{\Gamma(\beta-1)}{\Gamma(\beta-p-2)} t_{1}^{\beta-p-2}\right) \\
& \times J\left(t_{1}, x\left(t_{1}\right), D_{0^{+}}^{q} x\left(t_{1}\right)\right) \\
& +\frac{t^{\beta-1}\left(t_{1}^{\beta-2}-t_{1}^{\beta-1}\right)}{\Xi} J_{1}\left(t_{1}, x\left(t_{1}\right), D_{0^{+}}^{q} x\left(t_{1}\right)\right), \\
& \int_{0}^{t} \frac{(t-s)^{\beta-1}}{\Gamma(\beta)} \psi(s) g\left(s, x(s), D_{0^{+}}^{q} x(s)\right) d s \\
& \quad-\frac{t^{\beta-1}}{\Gamma(\beta)} \int_{0}^{1}(1-s)^{\beta-1} \psi(s) g\left(s, x(s), D_{0^{+}}^{q} x(s)\right) d s \\
& +\left(t^{\beta-2}-t^{\beta-1}\right) \int_{0}^{1} v(s) H\left(s, y(s), D_{0^{+}}^{p} y(s)\right) d s \\
& +t^{\beta-1} \int_{0}^{1} n(s) N\left(s, x(s), D_{0^{+}}^{q} x(s)\right) d s \\
& +\frac{t^{\beta-1}-t^{\beta-2}}{\Xi} \frac{\Gamma(\beta)}{\Gamma(\beta-p)} t_{1}^{\beta-p-1} \\
& \times J\left(t_{1}, x\left(t_{1}\right), D_{0^{+}}^{q} x\left(t_{1}\right)\right) \\
& +\frac{t^{\beta-2}-t^{\beta-1}}{\Xi} t_{1}^{\beta-1} J_{1}\left(t_{1}, x\left(t_{1}\right), D_{0^{+}}^{q} x\left(t_{1}\right)\right), \\
& t \in\left(t_{1}, 1\right] . \\
&
\end{aligned}
$$

Remark 9. By Lemmas 7 and $8,(x, y) \in X \times Y$ is a solution of BVP (6)-(7) if and only if $(x, y) \in X \times Y$ is a fixed point of the operator $T$.

Lemma 10. Suppose that (a)-(e) and (A)-(B) hold. Then $T$ : $X \times Y \rightarrow X \times Y$ is well defined and is completely continuous.

Proof. The proof is very long, so we list the steps. First, we prove that $T$ is well defined; second, we prove that $T$ is continuous, and, finally, we prove that $T$ is compact. So $T$ is completely continuous. Thus, the proof is divided into three steps.

Step 1. Prove that $T: X \times Y \rightarrow X \times Y$ is well defined.

For $(x, y) \in X \times Y$, we have $\|(x, y)\|=r>0$. Then

$$
\max \left\{\sup _{t \in(0,1)} t^{2-\alpha}|x(t)|, \sup _{t \in(0,1)} t^{2+q-\alpha}\left|D_{0^{+}}^{q} x(t)\right|\right\} \leq r<+\infty
$$

$$
\max \left\{\sup _{t \in(0,1)} t^{2-\beta}|y(t)|, \sup _{t \in(0,1)} t^{2+p-\beta}\left|D_{0^{+}}^{p} y(t)\right|\right\} \leq r<+\infty .
$$

From (B), $f, G, M, I, I_{1}$ are $\beta$-Caratheodory functions, then there exist constants $A_{r}>0$ such that

$$
\begin{gathered}
\left|f\left(t, y(t), D_{0^{+}}^{p} y(t)\right)\right| \leq A_{r}, \quad t \in(0,1), \\
\left|G\left(t, y(t), D_{0^{+}}^{p} y(t)\right)\right| \leq A_{r}, \quad t \in(0,1), \\
\left|M\left(t, y(t), D_{0^{+}}^{p} y(t)\right)\right| \leq A_{r}, \quad t \in(0,1), \\
\left|I\left(t_{1}, y\left(t_{1}\right), D_{0^{+}}^{p} y\left(t_{1}\right)\right)\right| \leq A_{r}, \quad t \in(0,1), \\
\left|I_{1}\left(t_{1}, y\left(t_{1}\right), D_{0^{+}}^{p} y\left(t_{1}\right)\right)\right| \leq A_{r}, \quad t \in(0,1) .
\end{gathered}
$$

Hence,

$$
\begin{aligned}
& \left|\int_{0}^{t} \frac{(t-s)^{\alpha-1}}{\Gamma(\alpha)} \phi(u) f\left(u, y(u), D_{0^{+}}^{p} y(u)\right) d u\right| \\
& \quad \leq \int_{0}^{t} \frac{(t-s)^{\alpha-1}}{\Gamma(\alpha)}\left|\phi(u) f\left(u, y(u), D_{0^{+}}^{p} y(u)\right)\right| d u \\
& \quad \leq A_{r} L_{1} \frac{\mathbf{B}(\alpha+\delta, k+1)}{\Gamma(\alpha)}<\infty .
\end{aligned}
$$

From (34), (37), and (38), we see that $\left(T_{1} y\right)(t)$ is defined on $(0,1]$, continuous on $\left(0, t_{1}\right]$ and $\left(t_{1}, 1\right]$, respectively. One sees that

$$
\begin{aligned}
& \lim _{t \rightarrow 0} t^{2-\alpha}\left(T_{1} y\right)(t) \\
&=\lim _{t \rightarrow 0}\left[t^{2-\alpha} \int_{0}^{t} \frac{(t-s)^{\alpha-1}}{\Gamma(\alpha)} \phi(u) f\left(u, y(u), D_{0^{+}}^{p} y(u)\right) d u\right. \\
&-\frac{t}{\Gamma(\alpha)} \int_{0}^{1}(1-s)^{\alpha-1} \phi(s) f\left(s, y(s), D_{0^{+}}^{p} y(s)\right) d s \\
&+\int_{0}^{1} u(s) G\left(s, y(s), D_{0^{+}}^{p} y(s)\right) d s \\
&+ \\
&+\int_{0}^{1} m(s) M\left(s, y(s), D_{0^{+}}^{p} y(s)\right) d s \\
&+\frac{t}{\Pi}\left(\frac{\Gamma(\alpha)}{\Gamma(\alpha-q)} t_{1}^{\alpha-q-1}-\frac{\Gamma(\alpha-1)}{\Gamma(\alpha-q-2)} t_{1}^{\alpha-q-2}\right) \\
& \times \\
& \quad I\left(t_{1}, y\left(t_{1}\right), D_{0^{+}}^{p} y\left(t_{1}\right)\right) \\
&\left.+\frac{t\left(t_{1}^{\alpha-2}-t_{1}^{\alpha-1}\right)}{\Pi} I_{1}\left(t_{1}, y\left(t_{1}\right), D_{0^{+}}^{p} y\left(t_{1}\right)\right)\right] \\
&=\int_{0}^{1} u(s) G\left(s, y(s), D_{0^{+}}^{p} y(s)\right) d s,
\end{aligned}
$$

and there exits the limit $\lim _{t \rightarrow t_{1}^{+}}\left(T_{1} y\right)(t)$. 
On the other hand, we have $D_{0^{+}}^{q}\left(T_{1} y\right)(t)$

$$
\begin{aligned}
& \iint_{0}^{t} \frac{(t-s)^{\alpha-q-1}}{\Gamma(\alpha-q)} \phi(u) f\left(u, y(u), D_{0^{+}}^{p} y(u)\right) d u \\
& -\frac{t^{\alpha-q-1}}{\Gamma(\alpha-q)} \\
& \times \int_{0}^{1}(1-s)^{\alpha-1} \phi(s) f\left(s, y(s), D_{0^{+}}^{p} y(s)\right) d s \\
& +t^{\alpha-q-2} \frac{\Gamma(\alpha-1)}{\Gamma(\alpha-q-1)} \\
& \times \int_{0}^{1} u(s) G\left(s, y(s), D_{0^{+}}^{p} y(s)\right) d s \\
& +t^{\alpha-q-1} \frac{\Gamma(\alpha)}{\Gamma(\alpha-q)} \\
& \times \int_{0}^{1} m(s) M\left(s, y(s), D_{0^{+}}^{p} y(s)\right) d s \\
& +\frac{t^{\alpha-q-1}}{\Pi} \frac{\Gamma(\alpha)}{\Gamma(\alpha-q)} \\
& \times\left(\frac{\Gamma(\alpha)}{\Gamma(\alpha-q)} t_{1}^{\alpha-q-1}-\frac{\Gamma(\alpha-1)}{\Gamma(\alpha-q-2)} t_{1}^{\alpha-q-2}\right) \\
& \times I\left(t_{1}, y\left(t_{1}\right), D_{0^{+}}^{p} y\left(t_{1}\right)\right) \\
& +\frac{\Gamma(\alpha)}{\Gamma(\alpha-q)} \frac{t^{\alpha-q-1}\left(t_{1}^{\alpha-2}-t_{1}^{\alpha-1}\right)}{\Pi} \\
& \times I_{1}\left(t_{1}, y\left(t_{1}\right), D_{0^{+}}^{p} y\left(t_{1}\right)\right), \quad t \in\left(0, t_{1}\right], \\
& \int_{0}^{t} \frac{(t-s)^{\alpha-q-1}}{\Gamma(\alpha-q)} \phi(s) f\left(s, y(s), D_{0^{+}}^{p} y(s)\right) d s \\
& -\frac{t^{\alpha-q-1}}{\Gamma(\alpha-q)} \\
& \times \int_{0}^{1}(1-s)^{\alpha-1} \phi(s) f\left(s, y(s), D_{0^{+}}^{p} y(s)\right) d s \\
& +\left(t^{\alpha-q-2} \frac{\Gamma(\alpha-1)}{\Gamma(\alpha-q-1)}-t^{\alpha-q-1} \frac{\Gamma(\alpha)}{\Gamma(\alpha-q)}\right) \\
& \times \int_{0}^{1} u(s) G\left(s, y(s), D_{0^{+}}^{p} y(s)\right) d s \\
& +t^{\alpha-q-1} \frac{\Gamma(\alpha)}{\Gamma(\alpha-q)} \int_{0}^{1} m(s) M\left(s, y(s), D_{0^{+}}^{p} y(s)\right) d s \\
& +\frac{1}{\Pi}\left(t^{\alpha-q-1} \frac{\Gamma(\alpha)}{\Gamma(\alpha-q)}-t^{\alpha-q-2} \frac{\Gamma(\alpha-1)}{\Gamma(\alpha-q-1)}\right) \\
& \times \frac{\Gamma(\alpha)}{\Gamma(\alpha-q)} t_{1}^{\alpha-q-1} I\left(t_{1}, y\left(t_{1}\right), D_{0^{+}}^{p} y\left(t_{1}\right)\right) \\
& +\frac{1}{\Pi}\left(t^{\alpha-q-2} \frac{\Gamma(\alpha-1)}{\Gamma(\alpha-q-1)}-t^{\alpha-q-1} \frac{\Gamma(\alpha)}{\Gamma(\alpha-q)}\right) \\
& \times t_{1}^{\alpha-1} I_{1}\left(t_{1}, y\left(t_{1}\right), D_{0^{+}}^{p} y\left(t_{1}\right)\right), \quad t \in\left(t_{1}, 1\right],
\end{aligned}
$$

$D_{0^{+}}^{p}\left(T_{2} x\right)(t)$

$$
\begin{aligned}
& \int_{0}^{t} \frac{(t-s)^{\beta-p-1}}{\Gamma(\beta-p)} \psi(u) g\left(u, x(u), D_{0^{+}}^{q} x(u)\right) d u \\
& -\frac{t^{\beta-p-1}}{\Gamma(\beta-p)} \\
& \times \int_{0}^{1}(1-s)^{\beta-1} \psi(s) g\left(s, x(s), D_{0^{+}}^{q} x(s)\right) d s \\
& +t^{\beta-p-2} \frac{\Gamma(\beta-1)}{\Gamma(\beta-p-1)} \\
& \times \int_{0}^{1} v(s) H\left(s, y(s), D_{0^{+}}^{p} y(s)\right) d s \\
& +t^{\beta-p-1} \frac{\Gamma(\beta)}{\Gamma(\beta-p)} \\
& \times \int_{0}^{1} n(s) N\left(s, x(s), D_{0^{+}}^{q} x(s)\right) d s \\
& +\frac{t^{\beta-p-1}}{\Xi} \frac{\Gamma(\beta)}{\Gamma(\beta-p)} \\
& \times\left(\frac{\Gamma(\beta)}{\Gamma(\beta-p)} t_{1}^{\beta-p-1}-\frac{\Gamma(\beta-1)}{\Gamma(\beta-p-2)} t_{1}^{\beta-p-2}\right) \\
& \times J\left(t_{1}, x\left(t_{1}\right), D_{0^{+}}^{q} x\left(t_{1}\right)\right) \\
& =\left\{+\frac{t^{\beta-p-1}\left(t_{1}^{\beta-2}-t_{1}^{\beta-1}\right)}{\Xi} \frac{\Gamma(\beta)}{\Gamma(\beta-p)}\right. \\
& \times J_{1}\left(t_{1}, x\left(t_{1}\right), D_{0^{+}}^{q} x\left(t_{1}\right)\right), \quad t \in\left(0, t_{1}\right], \\
& \int_{0}^{t} \frac{(t-s)^{\beta-p-1}}{\Gamma(\beta-p)} \psi(s) g\left(s, x(s), D_{0^{+}}^{q} x(s)\right) d s \\
& -\frac{t^{\beta-p-1}}{\Gamma(\beta)} \frac{\Gamma(\beta)}{\Gamma(\beta-p)} \\
& \times \int_{0}^{1}(1-s)^{\beta-1} \psi(s) g\left(s, x(s), D_{0^{+}}^{q} x(s)\right) d s \\
& +\left(t^{\beta-p-2} \frac{\Gamma(\beta-1)}{\Gamma(\beta-p-1)}-t^{\beta-p-1} \frac{\Gamma(\beta)}{\Gamma(\beta-p)}\right) \\
& \times \int_{0}^{1} v(s) H\left(s, y(s), D_{0^{+}}^{p} y(s)\right) d s \\
& +t^{\beta-p-1} \frac{\Gamma(\beta)}{\Gamma(\beta-p)} \int_{0}^{1} n(s) N\left(s, x(s), D_{0^{+}}^{q} x(s)\right) d s \\
& +\frac{1}{\Xi}\left(t^{\beta-p-1} \frac{\Gamma(\beta)}{\Gamma(\beta-p)}-t^{\beta-p-2} \frac{\Gamma(\beta-1)}{\Gamma(\beta-p-1)}\right) \\
& \times \frac{\Gamma(\beta)}{\Gamma(\beta-p)} t_{1}^{\beta-p-1} J\left(t_{1}, x\left(t_{1}\right), D_{0^{+}}^{q} x\left(t_{1}\right)\right) \\
& +\frac{1}{\Xi}\left(t^{\beta-p-2} \frac{\Gamma(\beta-1)}{\Gamma(\beta-p-1)}-t^{\beta-p-1} \frac{\Gamma(\beta)}{\Gamma(\beta-p)}\right) \\
& \times t_{1}^{\beta-1} J_{1}\left(t_{1}, x\left(t_{1}\right), D_{0^{+}}^{q} x\left(t_{1}\right)\right), \quad t \in\left(t_{1}, 1\right] \text {. }
\end{aligned}
$$


It is easy to see that

$$
\begin{gathered}
\left|\int_{0}^{t} \frac{(t-s)^{\alpha-q-1}}{\Gamma(\alpha-q)} \phi(u) f\left(u, y(u), D_{0^{+}}^{p} y(u)\right) d u\right| \\
\leq A_{r} L_{1} \frac{\mathbf{B}(\alpha+\delta-q, k+1)}{\Gamma(\alpha-q)}<\infty .
\end{gathered}
$$

From (37) and (41), we see that $D_{0^{+}}^{q}\left(T_{1} y\right)(t)$ is defined on $(0,1]$, continuous on $\left(0, t_{1}\right]$ and $\left(t_{1}, 1\right]$, respectively. One sees that

$$
\begin{aligned}
& \lim _{t \rightarrow 0} t^{2+q-\alpha} D_{0^{+}}^{q}\left(T_{1} y\right)(t) \\
& =\lim _{t \rightarrow 0}\left[t^{2+q-\alpha}\right. \\
& \times \int_{0}^{t} \frac{(t-s)^{\alpha-q-1}}{\Gamma(\alpha-q)} \phi(u) f\left(u, y(u), D_{0^{+}}^{p} y(u)\right) d u \\
& -\frac{t}{\Gamma(\alpha-q)} \\
& \times \int_{0}^{1}(1-s)^{\alpha-1} \phi(s) f\left(s, y(s), D_{0^{+}}^{p} y(s)\right) d s \\
& +\frac{\Gamma(\alpha-1)}{\Gamma(\alpha-q-1)} \int_{0}^{1} u(s) G\left(s, y(s), D_{0^{+}}^{p} y(s)\right) d s \\
& +t \frac{\Gamma(\alpha)}{\Gamma(\alpha-q)} \int_{0}^{1} m(s) M\left(s, y(s), D_{0^{+}}^{p} y(s)\right) d s \\
& +\frac{t}{\Pi} \frac{\Gamma(\alpha)}{\Gamma(\alpha-q)} \\
& \times\left(\frac{\Gamma(\alpha)}{\Gamma(\alpha-q)} t_{1}^{\alpha-q-1}-\frac{\Gamma(\alpha-1)}{\Gamma(\alpha-q-2)} t_{1}^{\alpha-q-2}\right) \\
& \times I\left(t_{1}, y\left(t_{1}\right), D_{0^{+}}^{p} y\left(t_{1}\right)\right) \\
& +\frac{\Gamma(\alpha)}{\Gamma(\alpha-q)} \frac{t\left(t_{1}^{\alpha-2}-t_{1}^{\alpha-1}\right)}{\Pi} \\
& \left.\times I_{1}\left(t_{1}, y\left(t_{1}\right), D_{0^{+}}^{p} y\left(t_{1}\right)\right)\right] \\
& =\frac{\Gamma(\alpha-1)}{\Gamma(\alpha-q-1)} \int_{0}^{1} u(s) G\left(s, y(s), D_{0^{+}}^{p} y(s)\right) d s,
\end{aligned}
$$

and there exits the $\operatorname{limit}_{t \rightarrow t_{1}^{+}} D_{0^{+}}^{q}\left(T_{1} y\right)(t)$.

From the above discussion, we have $\left(T_{1} y\right) \in X$. Similarly, we can show that $\left(T_{2} x\right) \in Y$. Hence, $\left(\left(T_{1} y\right),\left(T_{2} x\right)\right) \in X \times Y$. Then $T: X \times Y \rightarrow X \times Y$ is well defined.
Step 2. We prove that $T$ is continuous. Let $\left(x_{n}, y_{n}\right) \in X \times$ $Y$ with $\left(x_{n}, y_{n}\right) \rightarrow\left(x_{0}, y_{0}\right)$ as $n \rightarrow \infty$. We will show that $T\left(x_{n}, y_{n}\right) \rightarrow T\left(x_{0}, y_{0}\right)$ as $n \rightarrow \infty$, that is, prove that $T_{1} y_{n} \rightarrow$ $T_{1} y_{0}$ and $T_{2} x_{n} \rightarrow T_{2} x_{0}$ as $n \rightarrow \infty$.

In fact, we have $r>0$ such that $\left\|\left(x_{n}, y_{n}\right)\right\|=r>0$. Then

$$
\begin{aligned}
& \max \left\{\sup _{t \in(0,1)} t^{2-\alpha}\left|x_{n}(t)\right|, \sup _{t \in(0,1)} t^{2+q-\alpha}\left|D_{0^{+}}^{q} x_{n}(t)\right|\right\} \\
& \leq r<+\infty, \quad n=0,1,2, \ldots, \\
& \max \left\{\sup _{t \in(0,1)} t^{2-\beta}\left|y_{n}(t)\right|, \sup _{t \in(0,1)} t^{2+p-\beta}\left|D_{0^{+}}^{p} y_{n}(t)\right|\right\} \\
& \leq r<+\infty, \quad n=0,1,2, \ldots
\end{aligned}
$$

From (B), $f, G, M, I, I_{1}$ are $\beta$-Caratheodory functions, then there exist constants $A_{r}>0$ such that

$$
\begin{gathered}
\left|f\left(t, y_{n}(t), D_{0^{+}}^{p} y_{n}(t)\right)\right| \leq A_{r}, \\
t \in(0,1), \quad n=0,1,2, \ldots, \\
\left|G\left(t, y_{n}(t), D_{0^{+}}^{p} y_{n}(t)\right)\right| \leq A_{r}, \\
t \in(0,1), \quad n=0,1,2, \ldots, \\
\left|M\left(t, y_{n}(t), D_{0^{+}}^{p} y_{n}(t)\right)\right| \leq A_{r}, \\
t \in(0,1), \quad n=0,1,2, \ldots, \\
\left|I\left(t_{1}, y_{n}\left(t_{1}\right), D_{0^{+}}^{p} y_{n}\left(t_{1}\right)\right)\right| \leq A_{r}, \\
t \in(0,1), \quad n=0,1,2, \ldots, \\
\left|I_{1}\left(t_{1}, y_{n}\left(t_{1}\right), D_{0^{+}}^{p} y_{n}\left(t_{1}\right)\right)\right| \leq A_{r}, \\
t \in(0,1), \quad n=0,1,2, \ldots, \\
\sup _{t \in(0,1)} t^{2-\beta}\left|y_{n}(t)-y_{0}(t)\right|, \\
\sup _{t \in(0,1)} t^{2-\alpha}\left|x_{n}(t)-x_{0}(t)\right| \longrightarrow 0,
\end{gathered}
$$

$$
\begin{aligned}
& \sup _{t \in(0,1)} t^{2+q-\alpha}\left|D_{0^{+}}^{q} x_{n}(t)-D_{0^{+}}^{q} x_{0}(t)\right| \longrightarrow 0, \\
& \sup _{t \in(0,1)} t^{2+p-\beta}\left|D_{0^{+}}^{p} y_{n}(t)-D_{0^{+}}^{p} y_{0}(t)\right| \longrightarrow 0,
\end{aligned}
$$


as $n \rightarrow \infty$. We have

$D_{0^{+}}^{q}\left(T_{1} y_{n}\right)(t)$

$$
\begin{aligned}
& \iint_{0}^{t} \frac{(t-s)^{\alpha-q-1}}{\Gamma(\alpha-q)} \phi(u) f\left(u, y_{n}(u), D_{0^{+}}^{p} y_{n}(u)\right) d u \\
& -\frac{t^{\alpha-q-1}}{\Gamma(\alpha-q)} \\
& \times \int_{0}^{1}(1-s)^{\alpha-1} \phi(s) f\left(s, y_{n}(s), D_{0^{+}}^{p} y_{n}(s)\right) d s \\
& +t^{\alpha-q-2} \frac{\Gamma(\alpha-1)}{\Gamma(\alpha-q-1)} \\
& \times \int_{0}^{1} u(s) G\left(s, y_{n}(s), D_{0^{+}}^{p} y_{n}(s)\right) d s \\
& +t^{\alpha-q-1} \frac{\Gamma(\alpha)}{\Gamma(\alpha-q)} \\
& \times \int_{0}^{1} m(s) M\left(s, y_{n}(s), D_{0^{+}}^{p} y_{n}(s)\right) d s \\
& +\frac{t^{\alpha-q-1}}{\Pi} \frac{\Gamma(\alpha)}{\Gamma(\alpha-q)} \\
& \times\left(\frac{\Gamma(\alpha)}{\Gamma(\alpha-q)} t_{1}^{\alpha-q-1}-\frac{\Gamma(\alpha-1)}{\Gamma(\alpha-q-2)} t_{1}^{\alpha-q-2}\right) \\
& \times I\left(t_{1}, y_{n}\left(t_{1}\right), D_{0^{+}}^{p} y_{n}\left(t_{1}\right)\right) \\
& +\frac{\Gamma(\alpha)}{\Gamma(\alpha-q)} \frac{t^{\alpha-q-1}\left(t_{1}^{\alpha-2}-t_{1}^{\alpha-1}\right)}{\Pi} \\
& =\left\{\quad \times I_{1}\left(t_{1}, y_{n}\left(t_{1}\right), D_{0^{+}}^{p} y_{n}\left(t_{1}\right)\right), \quad t \in\left(0, t_{1}\right],\right. \\
& \int_{0}^{t} \frac{(t-s)^{\alpha-q-1}}{\Gamma(\alpha-q)} \phi(s) f\left(s, y_{n}(s), D_{0^{+}}^{p} y_{n}(s)\right) d s \\
& -\frac{t^{\alpha-q-1}}{\Gamma(\alpha-q)} \\
& \times \int_{0}^{1}(1-s)^{\alpha-1} \phi(s) f\left(s, y(s), D_{0^{+}}^{p} y(s)\right) d s \\
& +\left(t^{\alpha-q-2} \frac{\Gamma(\alpha-1)}{\Gamma(\alpha-q-1)}-t^{\alpha-q-1} \frac{\Gamma(\alpha)}{\Gamma(\alpha-q)}\right) \\
& \times \int_{0}^{1} u(s) G\left(s, y_{n}(s), D_{0^{+}}^{p} y_{n}(s)\right) d s \\
& +t^{\alpha-q-1} \frac{\Gamma(\alpha)}{\Gamma(\alpha-q)} \\
& \times \int_{0}^{1} m(s) M\left(s, y_{n}(s), D_{0^{+}}^{p} y_{n}(s)\right) d s \\
& +\frac{1}{\Pi}\left(t^{\alpha-q-1} \frac{\Gamma(\alpha)}{\Gamma(\alpha-q)}-t^{\alpha-q-2} \frac{\Gamma(\alpha-1)}{\Gamma(\alpha-q-1)}\right) \\
& \times \frac{\Gamma(\alpha)}{\Gamma(\alpha-q)} t_{1}^{\alpha-q-1} I\left(t_{1}, y_{n}\left(t_{1}\right), D_{0^{+}}^{p} y_{n}\left(t_{1}\right)\right) \\
& +\frac{1}{\Pi}\left(t^{\alpha-q-2} \frac{\Gamma(\alpha-1)}{\Gamma(\alpha-q-1)}-t^{\alpha-q-1} \frac{\Gamma(\alpha)}{\Gamma(\alpha-q)}\right) \\
& \times t_{1}^{\alpha-1} I_{1}\left(t_{1}, y_{n}\left(t_{1}\right), D_{0^{+}}^{p} y_{n}\left(t_{1}\right)\right), \quad t \in\left(t_{1}, 1\right] \text {. }
\end{aligned}
$$

From the Lebesgue dominated convergence theorem, we get

$$
\begin{gathered}
\sup _{t \in(0,1)} t^{2-\beta}\left|\left(T_{1} y_{n}\right)(t)-\left(T_{1} y_{0}\right)(t)\right|, \\
\sup _{t \in(0,1)} t^{2+p-\beta}\left|D_{0^{+}}^{p}\left(T_{1} y_{n}\right)(t)-D_{0^{+}}^{p}\left(T_{1} y_{0}\right)(t)\right| \longrightarrow 0,
\end{gathered}
$$

as $n \rightarrow \infty$. Similarly, we can show that

$$
\begin{gathered}
\sup _{t \in(0,1)} t^{2-\alpha}\left|\left(T_{2} x_{n}\right)(t)-\left(T_{2} x_{0}\right)(t)\right| \longrightarrow 0, \\
\sup _{t \in(0,1)} t^{2+q-\alpha}\left|D_{0^{+}}^{q}\left(T_{2} x_{n}\right)(t)-D_{0^{+}}^{q}\left(T_{2} x_{0}\right)(t)\right| \longrightarrow 0,
\end{gathered}
$$

as $n \rightarrow \infty$. It follows from (46) and (47) that $T$ is continuous.

Step 3. We prove that $T$ is compact, that is, for each nonempty open bounded subset $\Omega$ of $X \times Y$, prove that $T(\bar{\Omega})$ is relatively compact. We must prove that $T(\bar{\Omega})$ is uniformly bounded, equicontinuous on each subinterval $[a, b] \subseteq\left(t_{k}, t_{k+1}\right](k=$ $0,1), T(\bar{\Omega})$ is equiconvergent as $t \rightarrow 0$, and equiconvergent as $t \rightarrow t_{1}$.

Let $\Omega$ be a bounded open subset of $Y$. We have $r>0$ such that

$$
\begin{aligned}
\max & \left\{\sup _{t \in(0,1)} t^{2-\beta}|y(t)|, \sup _{t \in(0,1)} t^{2+p-\beta}\left|D_{0^{+}}^{p} y(t)\right|\right\} \\
& \leq r<+\infty, \quad y \in \bar{\Omega} .
\end{aligned}
$$

From (B), $f, G, M, I, I_{1}$ are $\beta$-Caratheodory functions, then there exist constants $A_{r}>0$ such that

$$
\begin{gathered}
\left|f\left(t, y(t), D_{0^{+}}^{p} y(t)\right)\right| \leq A_{r}, \quad t \in(0,1), \\
\left|G\left(t, y(t), D_{0^{+}}^{p} y(t)\right)\right| \leq A_{r}, \quad t \in(0,1), \\
\left|M\left(t, y(t), D_{0^{+}}^{p} y(t)\right)\right| \leq A_{r}, \quad t \in(0,1), \\
\left|I\left(t_{1}, y\left(t_{1}\right), D_{0^{+}}^{p} y\left(t_{1}\right)\right)\right| \leq A_{r}, \quad t \in(0,1), \\
\left|I_{1}\left(t_{1}, y\left(t_{1}\right), D_{0^{+}}^{p} y\left(t_{1}\right)\right)\right| \leq A_{r}, \quad t \in(0,1) .
\end{gathered}
$$

Substep 3.1. Prove that $T(\bar{\Omega})$ is uniformly bounded.

In fact, for $t \in\left(0, t_{1}\right]$, use (49), we have

$$
\begin{aligned}
& t^{2-\alpha}\left|\left(T_{1} y\right)(t)\right| \\
& \leq t^{2-\alpha} \int_{0}^{t} \frac{(t-s)^{\alpha-1}}{\Gamma(\alpha)}\left|\phi(u) f\left(u, y(u), D_{0^{+}}^{p} y(u)\right)\right| d u \\
& \quad+\frac{t}{\Gamma(\alpha)} \int_{0}^{1}(1-s)^{\alpha-1}\left|\phi(s) f\left(s, y(s), D_{0^{+}}^{p} y(s)\right)\right| d s \\
& \quad+\int_{0}^{1}\left|u(s) G\left(s, y(s), D_{0^{+}}^{p} y(s)\right)\right| d s \\
& \quad+t \int_{0}^{1}\left|m(s) M\left(s, y(s), D_{0^{+}}^{p} y(s)\right)\right| d s
\end{aligned}
$$




$$
\begin{aligned}
& +\frac{t}{\Pi}\left|\frac{\Gamma(\alpha)}{\Gamma(\alpha-q)} t_{1}^{\alpha-q-1}-\frac{\Gamma(\alpha-1)}{\Gamma(\alpha-q-2)} t_{1}^{\alpha-q-2}\right| \\
& \times\left|I\left(t_{1}, y\left(t_{1}\right), D_{0^{+}}^{p} y\left(t_{1}\right)\right)\right| \\
& +\frac{t\left(t_{1}^{\alpha-2}-t_{1}^{\alpha-1}\right)}{\Pi}\left|I_{1}\left(t_{1}, y\left(t_{1}\right), D_{0^{+}}^{p} y\left(t_{1}\right)\right)\right| \\
& \leq A_{r} L_{1} \frac{\mathbf{B}(\alpha+\delta, k+1)}{\Gamma(\alpha)}+\frac{A_{r} L_{1}}{\Gamma(\alpha)} \mathbf{B}(\alpha+\delta, k+1) \\
& +A_{r}\|u\|_{1}+A_{r}\|m\|_{1} \\
& +\frac{A_{r}}{\Pi}\left|\frac{\Gamma(\alpha)}{\Gamma(\alpha-q)} t_{1}^{\alpha-q-1}-\frac{\Gamma(\alpha-1)}{\Gamma(\alpha-q-2)} t_{1}^{\alpha-q-2}\right| \\
& +\frac{\left(t_{1}^{\alpha-2}-t_{1}^{\alpha-1}\right)}{\Pi} A_{r}<\infty .
\end{aligned}
$$

Similarly, we can get for $t \in\left(t_{1}, 1\right]$ that

$$
\begin{aligned}
& t^{2-\alpha}\left|\left(T_{1} y\right)(t)\right| \\
& \leq A_{r} L_{1} \frac{\mathbf{B}(\alpha+\delta, k+1)}{\Gamma(\alpha)}+\frac{A_{r} L_{1}}{\Gamma(\alpha)} \mathbf{B}(\alpha+\delta, k+1) \\
& +A_{r}\|u\|_{1}+A_{r}\|m\|_{1}+\frac{A_{r}}{\Pi} \frac{\Gamma(\alpha)}{\Gamma(\alpha-q)} t_{1}^{\alpha-q-1} \\
& +\frac{A_{r}}{\Pi} t_{1}^{\alpha-1}<\infty .
\end{aligned}
$$

Furthermore, we have for $t \in\left(0, t_{1}\right]$ that

$$
\begin{aligned}
& t^{2+q-\alpha}\left|D_{0^{+}}^{q}\left(T_{1} y\right)(t)\right| \\
& \leq A_{r} L_{1} \frac{\mathbf{B}(\alpha+\delta-q, k+1)}{\Gamma(\alpha-q)} \\
& \quad+\frac{A_{r} L_{1}}{\Gamma(\alpha-q)} \mathbf{B}(\alpha+\delta, k+1) \\
& \quad+\frac{A_{r} \Gamma(\alpha-1)}{\Gamma(\alpha-q-1)}\|u\|_{1}+\frac{A_{r} \Gamma(\alpha)}{\Gamma(\alpha-q)}\|m\|_{1} \\
& +\frac{A_{r}}{\Pi \frac{\Gamma(\alpha)}{\Gamma(\alpha-q)}} \\
& \quad \times\left|\frac{\Gamma(\alpha)}{\Gamma(\alpha-q)} t_{1}^{\alpha-q-1}-\frac{\Gamma(\alpha-1)}{\Gamma(\alpha-q-2)} t_{1}^{\alpha-q-2}\right| \\
& \quad+\frac{\Gamma(\alpha)}{\Gamma(\alpha-q)} \frac{A_{r}\left(t_{1}^{\alpha-2}-t_{1}^{\alpha-1}\right)}{\Pi}<\infty,
\end{aligned}
$$

and for $t \in\left(t_{1}, 1\right]$ that

$$
\begin{aligned}
t^{2+q-\alpha} & \left|D_{0^{+}}^{q}\left(T_{1} y\right)(t)\right| \\
\leq & A_{r} L_{1} \frac{\mathbf{B}(\alpha+\delta-q, k+1)}{\Gamma(\alpha-q)}+\frac{A_{r} L_{1}}{\Gamma(\alpha-q)} \mathbf{B}(\alpha+\delta, k+1) \\
& +\left(\frac{\Gamma(\alpha-1)}{\Gamma(\alpha-q-1)}+\frac{\Gamma(\alpha)}{\Gamma(\alpha-q)}\right) A_{r}\|u\|_{1} \\
& +\frac{\Gamma(\alpha)}{\Gamma(\alpha-q)} A_{r}\|m\|_{1} \\
& +\frac{1}{\Pi}\left(\frac{\Gamma(\alpha)}{\Gamma(\alpha-q)}+\frac{\Gamma(\alpha-1)}{\Gamma(\alpha-q-1)}\right) \frac{\Gamma(\alpha)}{\Gamma(\alpha-q)} t_{1}^{\alpha-q-1} A_{r} \\
& +\frac{1}{\Pi}\left(\frac{\Gamma(\alpha-1)}{\Gamma(\alpha-q-1)}+\frac{\Gamma(\alpha)}{\Gamma(\alpha-q)}\right) t_{1}^{\alpha-1} A_{r}<\infty .
\end{aligned}
$$

Hence,

$$
\begin{aligned}
\max & \left\{\sup _{t \in(0,1)} t^{2-\alpha}|(T y)(t)|, \sup _{t \in(0,1)} t^{2+q-\alpha}\left|D_{0^{+}}^{q}(T y)(t)\right|\right\} \\
& <+\infty, \quad y \in \bar{\Omega} .
\end{aligned}
$$

Similarly, we can show that

$$
\begin{aligned}
\max & \left\{\sup _{t \in(0,1)} t^{2-\beta}|(T x)(t)|, \sup _{t \in(0,1)} t^{2+p-\beta}\left|D_{0^{+}}^{p}(T x)(t)\right|\right\} \\
& <+\infty, \quad y \in \bar{\Omega} .
\end{aligned}
$$

It is easy to see that $T(\bar{\Omega})$ is uniformly bounded.

Substep 3.2. Prove that $T(\bar{\Omega})$ is equicontinuous on each subinterval $[a, b] \subseteq\left(t_{k}, t_{k+1}\right](k=0,1)$.

For each $[a, b] \subseteq\left(t_{0}, t_{1}\right]$, and $s_{1}, s_{2} \in[a, b]$ with $s_{2}<s_{1}$, we have

$$
\begin{aligned}
& \left|s_{1}^{2-\alpha}(T y)\left(s_{1}\right)-s_{2}^{2-\alpha}(T y)\left(s_{2}\right)\right| \\
& \leq \mid s_{1}^{2-\alpha} \int_{0}^{s_{1}} \frac{\left(s_{1}-s\right)^{\alpha-1}}{\Gamma(\alpha)} \phi(u) f\left(u, y(u), D_{0^{+}}^{p} y(u)\right) d u \\
& \quad-s_{2}^{2-\alpha} \int_{0}^{s_{2}} \frac{\left(s_{2}-s\right)^{\alpha-1}}{\Gamma(\alpha)} \phi(u) f\left(u, y(u), D_{0^{+}}^{p} y(u)\right) d u \mid \\
& \quad+\left|s_{1}-s_{2}\right| A_{r} \\
& \quad \times\left[\frac{L_{1} \mathbf{B}(\alpha+\delta, k+1)}{\Gamma(\alpha)}+\|m\|_{1}\right. \\
& \quad+\frac{1}{\Pi}\left|\frac{\Gamma(\alpha)}{\Gamma(\alpha-q)} t_{1}^{\alpha-q-1}-\frac{\Gamma(\alpha-1)}{\Gamma(\alpha-q-2)} t_{1}^{\alpha-q-2}\right| \\
& \left.+\frac{t_{1}^{\alpha-2}-t_{1}^{\alpha-1}}{\Pi}\right] .
\end{aligned}
$$

Note that $\left|\tau_{1}^{\varrho}-\tau_{2}^{\varrho}\right| \leq\left|\tau_{1}-\tau_{2}\right|^{\varrho}$ for all $\tau_{1}, \tau_{2} \geq 0$ and $\varrho \in(0,1)$. 
Since

$$
\begin{aligned}
& \mid s_{1}^{2-\alpha} \int_{0}^{s_{1}} \frac{\left(s_{1}-s\right)^{\alpha-1}}{\Gamma(\alpha)} \phi(u) f\left(u, y(u), D_{0^{+}}^{p} y(u)\right) d u \\
& -s_{2}^{2-\alpha} \int_{0}^{s_{2}} \frac{\left(s_{2}-s\right)^{\alpha-1}}{\Gamma(\alpha)} \phi(u) f\left(u, y(u), D_{0^{+}}^{p} y(u)\right) d u \mid \\
& \leq\left|s_{1}^{2-\alpha}-s_{2}^{2-\alpha}\right| A_{r} L_{1} \frac{\mathbf{B}(\alpha+\delta, k+1)}{\Gamma(\alpha)} \\
& +b^{2-\alpha} A_{r} L_{1} s_{1}^{\alpha+k+\delta} \int_{s_{2} / s_{1}}^{1} \frac{(1-w)^{\alpha+\delta-1} w^{k} d w}{\Gamma(\alpha)} \\
& +\left|s_{1}-s_{2}\right|^{\alpha-1} \frac{A_{r} L_{1} b^{2-\alpha}}{\Gamma(\alpha)} \int_{0}^{b} s^{k}(1-s)^{\delta} d s \longrightarrow 0 \\
& \text { uniformly as } s_{1} \longrightarrow s_{2} .
\end{aligned}
$$

It follows that

$$
\begin{array}{r}
\left|s_{1}^{2-\alpha}(T y)\left(s_{1}\right)-s_{2}^{2-\alpha}(T y)\left(s_{2}\right)\right| \longrightarrow 0 \\
\text { uniformly as } s_{1} \longrightarrow s_{2} .
\end{array}
$$

For $[a, b] \subseteq\left(t_{1}, 1\right]$, and $s_{1}, s_{2} \in[a, b]$ with $s_{2}<s_{1}$, we can prove similarly that

$$
\begin{array}{r}
\left|s_{1}^{2-\alpha}(T y)\left(s_{1}\right)-s_{2}^{2-\alpha}(T y x)\left(s_{2}\right)\right| \longrightarrow 0 \\
\text { uniformly as } s_{1} \longrightarrow s_{2} .
\end{array}
$$

On the other hand, for $[a, b] \subseteq\left(t_{0}, t_{1}\right]$, and $s_{1}, s_{2} \in[a, b]$ with $s_{2}<s_{1}$, we have

$$
\begin{aligned}
& \left|s_{1}^{2+q-\alpha} D_{0^{+}}^{q}\left(T_{1} y\right)\left(s_{1}\right)-s_{2}^{2+q-\alpha} D_{0^{+}}^{q}\left(T_{1} y\right)\left(s_{2}\right)\right| \\
& \leq \mid s_{1}^{2+q-\alpha} \int_{0}^{s_{1}} \frac{\left(s_{1}-s\right)^{\alpha-q-1}}{\Gamma(\alpha-q)} \phi(u) f\left(u, y(u), D_{0^{+}}^{p} y(u)\right) d u \\
& -s_{2}^{2+q-\alpha} \int_{0}^{s_{2}} \frac{\left(s_{2}-s\right)^{\alpha-q-1}}{\Gamma(\alpha-q)} \phi(u) f\left(u, y(u), D_{0^{+}}^{p} y(u)\right) d u \mid \\
& +\left|s_{2}-s_{1}\right| A_{r}\left[\frac{L_{1} B(\alpha+\delta, k+1)}{\Gamma(\alpha-q)}+\frac{\Gamma(\alpha)\|m\|_{1}}{\Gamma(\alpha-q)}\right. \\
& +\frac{1}{\Pi \frac{\Gamma(\alpha)}{\Gamma(\alpha-q)}} \\
& \times\left|\frac{\Gamma(\alpha)}{\Gamma(\alpha-q)} t_{1}^{\alpha-q-1}-\frac{\Gamma(\alpha-1)}{\Gamma(\alpha-q-2)} t_{1}^{\alpha-q-2}\right| \\
& +\frac{\Gamma(\alpha)}{\Gamma(\alpha-q)} \frac{\left|t_{1}^{\alpha-2}-t_{1}^{\alpha-1}\right|}{\Pi} \mid
\end{aligned}
$$

It is easy to see that

$$
\begin{gathered}
\left|s_{1}^{2+q-\alpha}-s_{2}^{2+q-\alpha}\right| A_{r} L_{1} \int_{0}^{1} \frac{(1-w)^{\alpha+\delta-q-1} w^{k} d s}{\Gamma(\alpha-q)} \longrightarrow 0 \\
\text { uniformly as } s_{1} \longrightarrow s_{2}, \\
b^{2+q-\alpha} A_{r} L_{1} \int_{s_{2} / s_{1}}^{1} \frac{(1-w)^{\alpha-q-1} w^{k} d w}{\Gamma(\alpha-q)} \longrightarrow 0 \\
\text { uniformly as } s_{1} \longrightarrow s_{2} .
\end{gathered}
$$

For the third term, if $\alpha-q-1 \geq 0$, use $\left|\tau_{1}^{\varrho}-\tau_{2}^{\varrho}\right| \leq\left|\tau_{1}-\tau_{2}\right|^{\varrho}$, then

$$
\begin{aligned}
& \int_{0}^{s_{2}} \frac{\left|\left(s_{1}-s\right)^{\alpha-q-1}-\left(s_{2}-s\right)^{\alpha-q-1}\right|}{\Gamma(\alpha-q)} s^{k}(1-s)^{\delta} d s \\
& \quad \leq\left|s_{1}-s_{2}\right|^{\alpha-q-1} \int_{0}^{b} \frac{1}{\Gamma(\alpha-q)} s^{k}(1-s)^{\delta} d s \longrightarrow 0
\end{aligned}
$$

$$
\text { uniformly as } s_{1} \longrightarrow s_{2} \text {. }
$$

If $\alpha-q-1<0$, use $\left|\tau_{1}^{\varrho}-\tau_{2}^{\varrho}\right| \leq\left|\tau_{1}-\tau_{2}\right|^{\varrho}$, then

$$
\begin{gathered}
\int_{0}^{s_{2}} \frac{\left|\left(s_{1}-s\right)^{\alpha-q-1}-\left(s_{2}-s\right)^{\alpha-q-1}\right|}{\Gamma(\alpha-q)} s^{k}(1-s)^{\delta} d s \\
=\int_{0}^{s_{2}} \frac{\left(s_{2}-s\right)^{\alpha-q-1}-\left(s_{1}-s\right)^{\alpha-q-1}}{\Gamma(\alpha-q)} s^{k}\left(s_{1}-s\right)^{\delta} d s \\
\leq\left|s_{2}^{\alpha+\delta+k-q}-s_{1}^{\alpha+\delta+k-q}\right| \frac{\mathbf{B}(\alpha+\delta+k-q, k+1)}{\gamma(\alpha-q)} \\
\quad+b^{\alpha+k+\delta-q} \frac{\mathbf{B}(\alpha+2 \delta-q, k+1)}{\Gamma(\alpha-q)}\left|1-\frac{s_{1}}{s_{2}}\right|^{-\delta} \longrightarrow 0 \\
\text { uniformly as } s_{1} \longrightarrow s_{2} .
\end{gathered}
$$

For $[a, b] \subseteq\left(t_{1}, 1\right]$, and $s_{1}, s_{2} \in[a, b]$ with $s_{2}<s_{1}$, we can prove similarly that

$$
\begin{array}{r}
\left|s_{1}^{2+q-\alpha} D_{0^{+}}^{q}\left(T_{1} y\right)\left(s_{1}\right)-s_{2}^{2+q-\alpha} D_{0^{+}}^{q}\left(T_{1} y\right)\left(s_{2}\right)\right| \\
\text { uniformly as } s_{1} \longrightarrow 0
\end{array}
$$

Similarly, we can show that for each $[a, b] \subseteq\left(t_{0}, t_{1}\right]$, and $s_{1}, s_{2} \in[a, b]$ with $s_{2}<s_{1}$, we have

$$
\begin{array}{r}
\left|s_{1}^{2-\beta}(T x)\left(s_{1}\right)-s_{2}^{2-\beta}(T x)\left(s_{2}\right)\right| \longrightarrow 0 \\
\text { uniformly as } s_{1} \longrightarrow s_{2} .
\end{array}
$$

For $[a, b] \subseteq\left(t_{1}, 1\right]$, and $s_{1}, s_{2} \in[a, b]$ with $s_{2}<s_{1}$, we can prove similarly that

$$
\begin{array}{r}
\left|s_{1}^{2-\beta}(T x)\left(s_{1}\right)-s_{2}^{2-\beta}(T x)\left(s_{2}\right)\right| \longrightarrow 0 \\
\text { uniformly as } s_{1} \longrightarrow s_{2} .
\end{array}
$$


For each $[a, b] \subseteq\left(t_{0}, t_{1}\right]$, and $s_{1}, s_{2} \in[a, b]$ with $s_{2}<s_{1}$, we have

$$
\begin{array}{r}
\left|s_{1}^{2+p-\beta} D_{0^{+}}^{p}(T x)\left(s_{1}\right)-s_{2}^{2+p-\beta} D_{0^{+}}^{p}(T x)\left(s_{2}\right)\right| \\
\text { uniformly as } s_{1} \longrightarrow 0
\end{array}
$$

For $[a, b] \subseteq\left(t_{1}, 1\right]$, and $s_{1}, s_{2} \in[a, b]$ with $s_{2}<s_{1}$, we can prove similarly that

$$
\begin{array}{r}
\left|s_{1}^{2+p-\beta} D_{0^{+}}^{p}(T x)\left(s_{1}\right)-s_{2}^{2+p-\beta} D_{0^{+}}^{p}(T x)\left(s_{2}\right)\right| \\
\text { uniformly as } s_{1} \longrightarrow 0
\end{array}
$$

So $T(\bar{\Omega})$ is equicontinuous on each subinterval $[a, b] \subseteq$ $\left(t_{k}, t_{k+1}\right](k=0,1)$.

Substep 3.3. Prove that $T(\bar{\Omega})$ is equiconvergent as $t \rightarrow 0$, and equiconvergent as $t \rightarrow t_{1}$.

We have

$$
\begin{aligned}
& \left|t^{2-\alpha}(T y)(t)-\int_{0}^{1} u(s) G\left(s, y(s), D_{0^{+}}^{p} y(s)\right) d s\right| \\
& \leq A_{r} L_{1} t^{2+k+\delta} \frac{\mathbf{B}(\alpha+\delta, k+1)}{\Gamma(\alpha)} \\
& \quad+\frac{t}{\Gamma(\alpha)} A_{r} L_{1} \mathbf{B}(\alpha+\delta, k+1) \\
& \quad+t A_{r}\|m\|_{1} \\
& \quad+\frac{t}{\Pi\left|\frac{\Gamma(\alpha)}{\Gamma(\alpha-q)} t_{1}^{\alpha-q-1}-\frac{\Gamma(\alpha-1)}{\Gamma(\alpha-q-2)} t_{1}^{\alpha-q-2}\right| A_{r}} \\
& \quad+\frac{t\left|t_{1}^{\alpha-2}-t_{1}^{\alpha-1}\right|}{\Pi} A_{r} .
\end{aligned}
$$

It follows that

$$
\left|t^{2-\alpha}(T y)(t)-\int_{0}^{1} u(s) G\left(s, y(s), D_{0^{+}}^{p} y(s)\right) d s\right| \longrightarrow 0
$$

uniformly as $t \longrightarrow 0$.

Similarly, we can show that $t^{2-\alpha}(T y)(t)$ is equiconvergent at $t=t_{1}$. On the other hand, we have

$$
\begin{aligned}
& \mid t^{2+q-\alpha} D_{0^{+}}^{q}(T y)(t)-\frac{\Gamma(\alpha-1)}{\Gamma(\alpha-q-1)} \\
& \times \int_{0}^{1} u(s) G\left(s, y(s), D_{0^{+}}^{p} y(s)\right) d s \mid \\
& \leq A_{r} L_{1} t^{2+k+\delta} \frac{\mathbf{B}(\alpha+\delta-q, k+1)}{\Gamma(\alpha-q)} \\
& \quad+\frac{t}{\Gamma(\alpha-q)} A_{r} L_{1} \mathbf{B}(\alpha+\delta, k+1) \\
& +t \frac{\Gamma(\alpha)}{\Gamma(\alpha-q)} A_{r}\|m\|_{1}+\frac{t}{\Pi} \frac{\Gamma(\alpha)}{\Gamma(\alpha-q)}
\end{aligned}
$$

$$
\begin{aligned}
& \times\left|\frac{\Gamma(\alpha)}{\Gamma(\alpha-q)} t_{1}^{\alpha-q-1}-\frac{\Gamma(\alpha-1)}{\Gamma(\alpha-q-2)} t_{1}^{\alpha-q-2}\right| A_{r} \\
& +\frac{\Gamma(\alpha)}{\Gamma(\alpha-q)} \frac{t\left|t_{1}^{\alpha-2}-t_{1}^{\alpha-1}\right|}{\Pi} A_{r} .
\end{aligned}
$$

It follows that

$$
\begin{array}{r}
\mid t^{2+q-\alpha} D_{0^{+}}^{q}(T y)(t)-\frac{\Gamma(\alpha-1)}{\Gamma(\alpha-q-1)} \\
\times \int_{0}^{1} u(s) G\left(s, y(s), D_{0^{+}}^{p} y(s)\right) d s \mid \rightarrow 0 \\
\text { uniformly as } t \longrightarrow 0 .
\end{array}
$$

Similarly, we can show that $t^{2+q-\alpha} D_{0^{+}}^{q}(T y)(t)$ is equiconvergent at $t=t_{1}$.

Similarly we can prove that

$$
\begin{array}{r}
\left|t^{2-\beta}(T x)(t)-\int_{0}^{1} v(s) H\left(s, x(s), D_{0^{+}}^{q} x(s)\right) d s\right| \\
\text { uniformly as } t \longrightarrow 0
\end{array}
$$

and $t^{2+p-\beta} D_{0^{+}}^{p}(T x)(t)$ is equiconvergent at $t=0$, both $t^{2-\beta}(T x)(t)$ and $t^{2+p-\beta} D_{0^{+}}^{p}(T x)(t)$ are equiconvergent at $t=$ $t_{1}$.

Hence, $T(\bar{\Omega})$ is equiconvergent as $t \rightarrow 0$ and $T(\bar{\Omega})$ is equiconvergent as $t \rightarrow t_{1}$.

So $T(\bar{\Omega})$ is relatively compact. Then $T$ is completely continuous. The proofs are completed.

\section{Main Result}

In this section, we will establish the existence of at least one solution of BVP (6)-(7).

Definition 11 (see [26]). An odd homeomorphism $\Phi$ of the real line $\mathbb{R}$ onto itself is called a pseudo-sub-multiplicative function if there exists a homeomorphism $\omega$ of $[0, \infty)$ onto itself which supports $\Phi$ in the sense that for all $v_{1}, v_{2} \geq 0$ we have $\Phi\left(v_{1} v_{2}\right) \geq \omega\left(v_{1}\right) \Phi\left(v_{2}\right)$. $\omega$ is called the supporting function of $\Phi$.

Remark 12. Note that any submultiplicative function is a pseudo-submultiplicative function. Also any function of the form $\Phi(u):=\sum_{j=0}^{k} c_{j}|u|^{j} u, u \in \mathbb{R}$ is pseudo-sup-multiplicative, provided that $c_{j} \geq 0$. Here, a supporting function is defined by $\omega(u):=\min \left\{u^{k+1}, u\right\}, u \geq 0$.

Remark 13. It is clear that a pseudo-submultiplicative function $\Phi$ and any corresponding supporting function $\omega$ are increasing functions vanishing at zero; moreover, their inverses $\Phi^{-1}$ and $\nu$, respectively, are increasing and for all $v_{1}, v_{2} \geq 0$, we have $\Phi^{-1}\left(v_{1} v_{2}\right) \leq v\left(v_{1}\right) \Phi^{-1}\left(v_{2}\right)$. 
Theorem 14. Suppose that (a)-(e) and (A)-(B) hold, $\Phi$ : $R \rightarrow R$ is a submultiplicative-like function with the supporting function $\omega$, its inverse function is denoted by $\Phi^{-1}: R \rightarrow R$ with the supporting function $v$. Furthermore, suppose that

(i) there exist nonnegative numbers $C_{f}, B_{f}, A_{f}, C_{G}, B_{G}$, $A_{G}, C_{M}, B_{M}$, and $A_{M}$ such that

$$
\begin{aligned}
& \left|f\left(t, t^{\alpha-2} U, t^{\alpha-q-2} V\right)\right| \\
& \quad \leq C_{f}+B_{f} \Phi^{-1}(|U|)+A_{f} \Phi^{-1}(|V|), \\
& \left|G\left(t, t^{\alpha-2} U, t^{\alpha-q-2} V\right)\right| \\
& \quad \leq C_{G}+B_{G} \Phi^{-1}(|U|)+A_{G} \Phi^{-1}(|V|), \\
& \left|M\left(t, t^{\alpha-2} U, t^{\alpha-q-2} V\right)\right| \\
& \quad \leq C_{M}+B_{M} \Phi^{-1}(|U|)+A_{M} \Phi^{-1}\left(\left|\Phi^{-1}(|U|)\right|\right),
\end{aligned}
$$

holds for all $(U, V) \in R^{2}, t \in(0,1]$.

(ii) there exist nonnegative numbers $C_{g}, B_{g}, A_{g}, C_{H}, B_{H}$, $A_{H}, C_{N}, B_{N}$, and $A_{N}$ such that

$$
\left|g\left(t, t^{\beta-2} U, t^{\beta-p-2} V\right)\right| \leq C_{g}+B_{g} \Phi(U)+A_{g} \Phi(V),
$$$$
\left|H\left(t, t^{\beta-2} U, t^{\beta-p-2} V\right)\right| \leq C_{H}+B_{H} \Phi(U)+A_{H} \Phi(V),
$$$$
\left|N\left(t, t^{\beta-2} U, t^{\beta-p-2} V\right)\right| \leq C_{N}+B_{N} \Phi(U)+A_{N} \Phi(V),
$$

hold for all $(U, V) \in R^{2}, t \in(0,1]$.

(iii) there exist the nonnegative numbers $C_{I}, B_{I}, A_{I}, C_{1, I}$, $B_{1, I}$, and $A_{1, I}$ such that

$$
\begin{gathered}
\left|I\left(t_{1}, t_{1}^{\alpha-2} U, t_{1}^{\alpha-q-2} V\right)\right| \leq C_{I}+B_{I} \Phi^{-1}(|U|)+A_{I} \Phi^{-1}(|V|), \\
\left|I_{1}\left(t_{1}, t_{1}^{\alpha-2} U, t_{1}^{\alpha-q-2} V\right)\right| \\
\leq C_{1, I}+B_{1, I} \Phi^{-1}(|U|)+A_{1, I} \Phi^{-1}(|V|)
\end{gathered}
$$

hold for all $(U, V) \in R^{2}$.

(iv) there exist the nonnegative numbers $C_{J}, B_{J}, A_{J}, C_{1, J}$, $B_{1, J}$, and $A_{1, J}$ such that

$$
\begin{gathered}
\left|J\left(t_{1}, t_{1}^{\beta-2} U, t_{1}^{\beta-p-2} V\right)\right| \leq C_{J}+B_{J} \Phi(U)+A_{J} \Phi(V), \\
\left|J_{1}\left(t_{1}, t_{1}^{\beta-2} U, t_{1}^{\beta-p-2} V\right)\right| \leq C_{1, J}+B_{1, J} \Phi(U)+A_{1, J} \Phi(V),
\end{gathered}
$$

hold for all $(U, V) \in R^{2}$.

Then BVP (6)-(7) has at least one solution if $\max \left\{\Theta_{2}, \Sigma_{2}, \Theta_{4}, \Sigma_{4}\right\} \nu\left(2 \max \left\{\Upsilon_{2}, \Lambda_{2}, \Upsilon_{4}, \Lambda_{4}\right\}\right)<1$ or $\frac{\max \left\{\Upsilon_{2}, \Lambda_{2}, \Upsilon_{4}, \Lambda_{4}\right\}}{w\left(\left(2 \max \left\{\Theta_{2}, \Sigma_{2}, \Theta_{4}, \Sigma_{4}\right\}\right)^{-1}\right)}<1$, where

$$
\begin{aligned}
& \Theta_{1}=L_{1} \frac{\mathbf{B}(\alpha+\delta, k+1)}{\Gamma(\alpha)} C_{f}+\frac{L_{1} \mathbf{B}(\alpha+\delta, k+1)}{\Gamma(\alpha)} C_{f} \\
& +\|u\|_{1} C_{G}+\|m\|_{1} C_{M} \\
& +\frac{1}{\Pi}\left|\frac{\Gamma(\alpha)}{\Gamma(\alpha-q)} t_{1}^{\alpha-q-1}-\frac{\Gamma(\alpha-1)}{\Gamma(\alpha-q-2)} t_{1}^{\alpha-q-2}\right| C_{I} \\
& +\frac{\left|t_{1}^{\alpha-2}-t_{1}^{\alpha-1}\right|}{\Pi} C_{1, I} \\
& \Theta_{2}=L_{1} \frac{\mathbf{B}(\alpha+\delta, k+1)}{\Gamma(\alpha)}\left[B_{f}+A_{f}\right] \\
& +\frac{L_{1} \mathbf{B}(\alpha+\delta, k+1)}{\Gamma(\alpha)}\left[B_{f}+A_{f}\right] \\
& +\|u\|_{1}\left[B_{G}+A_{G}\right]+\|m\|_{1}\left[B_{M}+A_{M}\right] \\
& +\frac{1}{\Pi}\left|\frac{\Gamma(\alpha)}{\Gamma(\alpha-q)} t_{1}^{\alpha-q-1}-\frac{\Gamma(\alpha-1)}{\Gamma(\alpha-q-2)} t_{1}^{\alpha-q-2}\right| \\
& \times\left[B_{I}+A_{I}\right]+\frac{\left|t_{1}^{\alpha-2}-t_{1}^{\alpha-1}\right|}{\Pi}\left[B_{1, I}+A_{1, I}\right], \\
& \Theta_{3}=L_{1} \frac{\mathbf{B}(\alpha+\delta-q, k+1)}{\Gamma(\alpha-q)} C_{f} \\
& +\frac{L_{1}}{\Gamma(\alpha-q)} \mathbf{B}(\alpha+\delta, k+1) C_{f} \\
& +\frac{\Gamma(\alpha-1)}{\Gamma(\alpha-q-1)}\|u\|_{1} C_{G}+\frac{\Gamma(\alpha)}{\Gamma(\alpha-q)}\|m\|_{1} C_{M} \\
& +\frac{1}{\Pi} \frac{\Gamma(\alpha)}{\Gamma(\alpha-q)} \\
& \times\left|\frac{\Gamma(\alpha)}{\Gamma(\alpha-q)} t_{1}^{\alpha-q-1}-\frac{\Gamma(\alpha-1)}{\Gamma(\alpha-q-2)} t_{1}^{\alpha-q-2}\right| C_{I} \\
& +\frac{\Gamma(\alpha)}{\Gamma(\alpha-q)} \frac{\left|t_{1}^{\alpha-2}-t_{1}^{\alpha-1}\right|}{\Pi} C_{1, I} \\
& \Theta_{4}=L_{1} \frac{\mathbf{B}(\alpha+\delta-q, k+1)}{\Gamma(\alpha-q)}\left[B_{f}+A_{f}\right] \\
& +\frac{L_{1}}{\Gamma(\alpha-q)} \mathbf{B}(\alpha+\delta, k+1)\left[B_{f}+A_{f}\right] \\
& +\frac{\Gamma(\alpha-1)}{\Gamma(\alpha-q-1)}\|u\|_{1}\left[B_{G}+A_{G}\right] \\
& +\frac{\Gamma(\alpha)}{\Gamma(\alpha-q)}\|m\|_{1}\left[B_{M}+A_{M}\right] \\
& +\frac{1}{\Pi} \frac{\Gamma(\alpha)}{\Gamma(\alpha-q)}
\end{aligned}
$$




$$
\begin{aligned}
& \times\left|\frac{\Gamma(\alpha)}{\Gamma(\alpha-q)} t_{1}^{\alpha-q-1}-\frac{\Gamma(\alpha-1)}{\Gamma(\alpha-q-2)} t_{1}^{\alpha-q-2}\right| \\
& +\frac{1}{\Pi}\left(\frac{\Gamma(\alpha-1)}{\Gamma(\alpha-q-1)}+\frac{\Gamma(\alpha)}{\Gamma(\alpha-q)}\right) \\
& \times\left[B_{I}+A_{I}\right] \\
& \times t_{1}^{\alpha-1}\left[B_{1, I}+A_{1, I}\right] \text {, } \\
& +\frac{\Gamma(\alpha)}{\Gamma(\alpha-q)} \frac{\left|t_{1}^{\alpha-2}-t_{1}^{\alpha-1}\right|}{\Pi}\left[B_{1, I}+A_{1, I}\right] \\
& \Sigma_{1}=L_{1} \frac{\mathbf{B}(\alpha+\delta, k+1)}{\Gamma(\alpha)} C_{f}+\frac{L_{1} \mathbf{B}(\alpha+\delta, k+1)}{\Gamma(\alpha)} C_{f} \\
& +\|u\|_{1} C_{G}+\frac{1}{\Pi} \frac{\Gamma(\alpha)}{\Gamma(\alpha-q)} t_{1}^{\alpha-q-1} C_{I}+\frac{1}{\Pi} t_{1}^{\alpha-1} C_{1, I}, \\
& \Sigma_{2}=L_{1} \frac{\mathbf{B}(\alpha+\delta, k+1)}{\Gamma(\alpha)}\left[B_{f}+A_{f}\right] \\
& +\frac{L_{1} \mathbf{B}(\alpha+\delta, k+1)}{\Gamma(\alpha)}\left[B_{f}+A_{f}\right]+\|u\|_{1}\left[B_{G}+A_{G}\right] \\
& +\frac{1}{\Pi} \frac{\Gamma(\alpha)}{\Gamma(\alpha-q)} t_{1}^{\alpha-q-1}\left[B_{I}+A_{I}\right]+\frac{1}{\Pi} t_{1}^{\alpha-1}\left[B_{1, I}+A_{1, I}\right], \\
& \Sigma_{3}=L_{1} \frac{\mathbf{B}(\alpha+\delta-q, k+1)}{\Gamma(\alpha-q)} C_{f} \\
& +\frac{L_{1}}{\Gamma(\alpha-q)} \mathbf{B}(\alpha+\delta, k+1) C_{f} \\
& +\left(\frac{\Gamma(\alpha-1)}{\Gamma(\alpha-q-1)}+\frac{\Gamma(\alpha)}{\Gamma(\alpha-q)}\right)\|u\|_{1} C_{G} \\
& +\frac{\Gamma(\alpha)}{\Gamma(\alpha-q)}\|m\|_{1} C_{M} \\
& +\frac{1}{\Pi}\left(\frac{\Gamma(\alpha)}{\Gamma(\alpha-q)}+\frac{\Gamma(\alpha-1)}{\Gamma(\alpha-q-1)}\right) \frac{\Gamma(\alpha)}{\Gamma(\alpha-q)} t_{1}^{\alpha-q-1} C_{I} \\
& +\frac{1}{\Pi}\left(\frac{\Gamma(\alpha-1)}{\Gamma(\alpha-q-1)}+\frac{\Gamma(\alpha)}{\Gamma(\alpha-q)}\right) t_{1}^{\alpha-1} C_{1, I}, \\
& \Sigma_{4}=L_{1} \frac{\mathbf{B}(\alpha+\delta-q, k+1)}{\Gamma(\alpha-q)}\left[B_{f}+A_{f}\right] \\
& +\frac{L_{1}}{\Gamma(\alpha-q)} \mathbf{B}(\alpha+\delta, k+1)\left[B_{f}+A_{f}\right] \\
& +\left(\frac{\Gamma(\alpha-1)}{\Gamma(\alpha-q-1)}+\frac{\Gamma(\alpha)}{\Gamma(\alpha-q)}\right)\|u\|_{1}\left[B_{G}+A_{G}\right] \\
& +\frac{\Gamma(\alpha)}{\Gamma(\alpha-q)}\|m\|_{1}\left[B_{M}+A_{M}\right] \\
& +\frac{1}{\Pi}\left(\frac{\Gamma(\alpha)}{\Gamma(\alpha-q)}+\frac{\Gamma(\alpha-1)}{\Gamma(\alpha-q-1)}\right) \\
& \times \frac{\Gamma(\alpha)}{\Gamma(\alpha-q)} t_{1}^{\alpha-q-1}\left[B_{I}+A_{I}\right] \\
& \Upsilon_{1}=L_{2} \frac{\mathbf{B}(\beta+\theta, l+1)}{\Gamma(\beta)} C_{g} \\
& +\frac{L_{2} \mathbf{B}(\beta+\theta, l+1)}{\Gamma(\beta)} C_{g}+\|v\|_{1} C_{H}+\|n\|_{1} C_{N} \\
& +\frac{1}{\Xi}\left|\frac{\Gamma(\beta)}{\Gamma(\beta-p)} t_{1}^{\beta-p-1}-\frac{\Gamma(\beta-1)}{\Gamma(\beta-p-2)} t_{1}^{\beta-p-2}\right| C_{J} \\
& +\frac{\left|t_{1}^{\beta-2}-t_{1}^{\beta-1}\right|}{\Xi} C_{1, J}, \\
& \Upsilon_{2}=L_{2} \frac{\mathbf{B}(\beta+\theta, l+1)}{\Gamma(\beta)}\left[B_{g}+A_{g}\right] \\
& +\frac{L_{2} \mathbf{B}(\beta+\theta, l+1)}{\Gamma(\beta)}\left[B_{g}+A_{g}\right] \\
& +\|b\|_{1}\left[B_{H}+A_{H}\right]+\|n\|_{1}\left[B_{N}+A_{N}\right] \\
& +\frac{1}{\Xi}\left|\frac{\Gamma(\beta)}{\Gamma(\beta-p)} t_{1}^{\beta-p-1}-\frac{\Gamma(\beta-1)}{\Gamma(\beta-p-2)} t_{1}^{\beta-p-2}\right| \\
& \times\left[B_{J}+A_{J}\right]+\frac{\left|t_{1}^{\beta-2}-t_{1}^{\beta-1}\right|}{\Xi}\left[B_{1, J}+A_{1, J}\right], \\
& \Upsilon_{3}=L_{2} \frac{\mathbf{B}(\beta+\theta-p, l+1)}{\Gamma(\beta-p)} C_{g} \\
& +\frac{L_{2}}{\Gamma(\beta-p)} \mathbf{B}(\beta+\theta, l+1) C_{g} \\
& +\frac{\Gamma(\beta-1)}{\Gamma(\beta-p-1)}\|v\|_{1} C_{H}+\frac{\Gamma(\beta)}{\Gamma(\beta-p)}\|n\|_{1} C_{N} \\
& +\frac{1}{\Xi} \frac{\Gamma(\beta)}{\Gamma(\beta-p)} \\
& \times\left|\frac{\Gamma(\beta)}{\Gamma(\beta-p)} t_{1}^{\beta-p-1}-\frac{\Gamma(\beta-1)}{\Gamma(\beta-p-2)} t_{1}^{\beta-p-2}\right| C_{J} \\
& +\frac{\Gamma(\beta)}{\Gamma(\beta-p)} \frac{\left|t_{1}^{\beta-2}-t_{1}^{\beta-1}\right|}{\Xi} C_{1, J}, \\
& \Upsilon_{4}=L_{2} \frac{\mathbf{B}(\beta+\theta-p, l+1)}{\Gamma(\beta-p)}\left[B_{g}+A_{g}\right] \\
& +\frac{L_{2}}{\Gamma(\beta-p)} \mathbf{B}(\beta+\theta, l+1)\left[B_{g}+A_{g}\right] \\
& +\frac{\Gamma(\beta-1)}{\Gamma(\beta-p-1)}\|v\|_{1}\left[B_{H}+A_{H}\right]
\end{aligned}
$$




$$
\begin{aligned}
& +\frac{\Gamma(\beta)}{\Gamma(\beta-p)}\|n\|_{1}\left[B_{N}+A_{N}\right] \\
& +\frac{1}{\Xi} \frac{\Gamma(\beta)}{\Gamma(\beta-p)} \\
& \times\left|\frac{\Gamma(\beta)}{\Gamma(\beta-p)} t_{1}^{\beta-p-1}-\frac{\Gamma(\beta-1)}{\Gamma(\beta-p-2)} t_{1}^{\beta-p-2}\right|\left[B_{J}+A_{J}\right] \\
& +\frac{\Gamma(\beta)}{\Gamma(\beta-p)} \frac{\left|t_{1}^{\beta-2}-t_{1}^{\beta-1}\right|}{\Xi}\left[B_{1, J}+A_{1, J}\right] \\
& \Lambda_{1}=L_{2} \frac{\mathbf{B}(\beta+\theta, l+1)}{\Gamma(\beta)} C_{g}+\frac{L_{2} \mathbf{B}(\beta+\theta, l+1)}{\Gamma(\beta)} C_{g} \\
& +\|v\|_{1} C_{H}+\frac{1}{\Xi} \frac{\Gamma(\beta)}{\Gamma(\beta-p)} t_{1}^{\beta-p-1} C_{J}+\frac{1}{\Xi} t_{1}^{\beta-1} C_{1, J}, \\
& \Lambda_{2}=L_{2} \frac{\mathbf{B}(\beta+\theta, l+1)}{\Gamma(\beta)}\left[B_{g}+A_{g}\right] \\
& +\frac{L_{2} \mathbf{B}(\beta+\theta, l+1)}{\Gamma(\beta)}\left[B_{g}+A_{g}\right]+\|v\|_{1}\left[B_{H}+A_{H}\right] \\
& +\frac{1}{\Xi} \frac{\Gamma(\beta)}{\Gamma(\beta-p)} t_{1}^{\beta-p-1}\left[B_{J}+A_{J}\right]+\frac{1}{\Xi} t_{1}^{\beta-1}\left[B_{1, J}+A_{1, J}\right], \\
& \Lambda_{3}=L_{2} \frac{\mathbf{B}(\beta+\theta-p, l+1)}{\Gamma(\beta-p)} C_{g} \\
& +\frac{L_{2}}{\Gamma(\beta-p)} \mathbf{B}(\beta+\theta, l+1) C_{g} \\
& +\left(\frac{\Gamma(\beta-1)}{\Gamma(\beta-p-1)}+\frac{\Gamma(\beta)}{\Gamma(\beta-p)}\right)\|v\|_{1} C_{H} \\
& +\frac{\Gamma(\beta)}{\Gamma(\beta-p)}\|n\|_{1} C_{N} \\
& +\frac{1}{\Xi}\left(\frac{\Gamma(\beta)}{\Gamma(\beta-p)}+\frac{\Gamma(\beta-1)}{\Gamma(\beta-p-1)}\right) \frac{\Gamma(\beta)}{\Gamma(\beta-p)} t_{1}^{\beta-p-1} C_{J} \\
& +\frac{1}{\Xi}\left(\frac{\Gamma(\beta-1)}{\Gamma(\beta-p-1)}+\frac{\Gamma(\beta)}{\Gamma(\beta-p)}\right) t_{1}^{\beta-1} C_{1, J}, \\
& \Lambda_{4}=L_{2} \frac{\mathbf{B}(\beta+\theta-p, l+1)}{\Gamma(\beta-p)}\left[B_{g}+A_{g}\right] \\
& +\frac{L_{2}}{\Gamma(\beta-p)} \mathbf{B}(\beta+\theta, l+1)\left[B_{g}+A_{g}\right] \\
& +\left(\frac{\Gamma(\beta-1)}{\Gamma(\beta-p-1)}+\frac{\Gamma(\beta)}{\Gamma(\beta-p)}\right)\|v\|_{1}\left[B_{H}+A_{H}\right] \\
& +\frac{\Gamma(\beta)}{\Gamma(\beta-p)}\|n\|_{1}\left[B_{N}+A_{N}\right]
\end{aligned}
$$$$
+\frac{1}{\Xi}\left(\frac{\Gamma(\beta)}{\Gamma(\beta-p)}+\frac{\Gamma(\beta-1)}{\Gamma(\beta-p-1)}\right)
$$$$
\begin{aligned}
& \times \frac{\Gamma(\beta)}{\Gamma(\beta-p)} t_{1}^{\beta-p-1}\left[B_{J}+A_{J}\right] \\
& +\frac{1}{\Xi}\left(\frac{\Gamma(\beta-1)}{\Gamma(\beta-p-1)}+\frac{\Gamma(\beta)}{\Gamma(\beta-p)}\right) t_{1}^{\beta-1}\left[B_{1, J}+A_{1, J}\right] .
\end{aligned}
$$

Proof. To apply Lemma 5, we should define an open bounded subset $\Omega$ of $X \times Y$ centered at zero such that assumptions in Lemma 5 hold.

Let $\Omega_{1}=\{(x, y) \in X \times Y:(x, y)=\lambda T(x, y)$ for some $\lambda \in(0,1)\}$. We prove that $\Omega_{1}$ is bounded. For $(x, y) \in \Omega_{1}$, we get $(x, y)=\lambda T(x, y)$. It follows that $x=\lambda T_{1} y$ and $y=\lambda T_{2} x$.

For $t \in\left(0, t_{1}\right]$, we obtain $t^{2-\alpha}|x(t)| \leq t^{2-\alpha}\left|\left(T_{1} y\right)(t)\right| \leq$ $\Theta_{1}+\Theta_{2} \Phi^{-1}(\|y\|)$.

For $t \in\left(t_{1}, 1\right]$,

$$
\begin{aligned}
t^{2-\alpha}|x(t)| & t^{2-\alpha} \int_{0}^{t} \frac{(t-s)^{\alpha-1}}{\Gamma(\alpha)}\left|\phi(s) f\left(s, y(s), D_{0^{+}}^{p} y(s)\right)\right| d s \\
& +\frac{t}{\Gamma(\alpha)} \int_{0}^{1}(1-s)^{\alpha-1}\left|\phi(s) f\left(s, y(s), D_{0^{+}}^{p} y(s)\right)\right| d s \\
& +(1-t) \int_{0}^{1}\left|u(s) G\left(s, y(s), D_{0^{+}}^{p} y(s)\right)\right| d s \\
& +t \int_{0}^{1}\left|m(s) M\left(s, y(s), D_{0^{+}}^{p} y(s)\right)\right| d s \\
& +\frac{1-t}{\Pi} \frac{\Gamma(\alpha)}{\Gamma(\alpha-q)} t_{1}^{\alpha-q-1}\left|I\left(t_{1}, y\left(t_{1}\right), D_{0^{+}}^{p} y\left(t_{1}\right)\right)\right| \\
& +\frac{1-t}{\Pi} t_{1}^{\alpha-1}\left|I_{1}\left(t_{1}, y\left(t_{1}\right), D_{0^{+}}^{p} y\left(t_{1}\right)\right)\right| \\
\leq & \Sigma_{1}+\Sigma_{2} \Phi^{-1}(\|y\|) .
\end{aligned}
$$

It follows that

$$
\sup _{t \in(0,1)} t^{2-\alpha}|x(t)| \leq \max \left\{\Theta_{1}, \Sigma_{1}\right\}+\max \left\{\Theta_{2}, \Sigma_{2}\right\} \Phi^{-1}(\|y\|) \text {. }
$$

Similarly, we have for $t \in\left(0, t_{1}\right]$ that

$$
t^{q+2-\alpha}\left|D_{0^{+}}^{q} x(t)\right| \leq \Theta_{3}+\Theta_{4} \Phi^{-1}(\|y\|)
$$

and for $t \in\left(0, t_{1}\right]$

$$
t^{q+2-\alpha}\left|D_{0^{+}}^{q} x(t)\right| \leq \Sigma_{3}+\Sigma_{4} \Phi^{-1}(\|y\|) .
$$

It follows that

$$
\begin{aligned}
& \sup _{t \in(0,1)} t^{2+q-\alpha}\left|D_{0^{+}}^{q} x(t)\right| \\
& \quad \leq \max \left\{\Theta_{3}, \Sigma_{3}\right\}+\max \left\{\Theta_{4}, \Sigma_{4}\right\} \Phi^{-1}(\|y\|) .
\end{aligned}
$$


Hence,

$$
\begin{aligned}
\|x\| \leq & \max \left\{\Theta_{1}, \Sigma_{1}, \Theta_{3}, \Sigma_{3}\right\} \\
& +\max \left\{\Theta_{2}, \Sigma_{2}, \Theta_{4}, \Sigma_{4}\right\} \Phi^{-1}(\|y\|) .
\end{aligned}
$$

Similar to the above discussion we can prove that

$\|y\| \leq \max \left\{\Upsilon_{1}, \Lambda_{1}, \Upsilon_{3}, \Lambda_{3}\right\}+\max \left\{\Upsilon_{2}, \Lambda_{2}, \Upsilon_{4}, \Lambda_{4}\right\} \Phi(\|x\|)$.

Case 1. Consider $\left(\max \left\{\Theta_{2}, \Sigma_{2}, \Theta_{4}, \Sigma_{4}\right\} \nu\left(2 \max \left\{\Upsilon_{2}, \Lambda_{2}, \Upsilon_{4}\right.\right.\right.$, $\left.\left.\left.\Lambda_{4}\right\}\right)<1\right)$.

With out loss of generality, suppose that

$$
\|x\| \geq \Phi^{-1}\left(\frac{\max \left\{\Upsilon_{1}, \Lambda_{1}, \Upsilon_{3}, \Lambda_{3}\right\}}{\max \left\{\Upsilon_{2}, \Lambda_{2}, \Upsilon_{4}, \Lambda_{4}\right\}}\right) .
$$

Then use Remark 13, and the previous inequalities to get

$$
\begin{aligned}
\|x\| \leq & \max \left\{\Theta_{1}, \Sigma_{1}, \Theta_{3}, \Sigma_{3}\right\} \\
& +\max \left\{\Theta_{2}, \Sigma_{2}, \Theta_{4}, \Sigma_{4}\right\} \nu\left(2 \max \left\{\Upsilon_{2}, \Lambda_{2}, \Upsilon_{4}, \Lambda_{4}\right\}\right)\|x\| .
\end{aligned}
$$

It follows that there exists a constant $W>0$ such that $\|x\| \leq$ $W$. Thus

$$
\|x\| \leq \max \left\{W, \Phi^{-1}\left(\frac{\max \left\{\Upsilon_{1}, \Lambda_{1}, \Upsilon_{3}, \Lambda_{3}\right\}}{\max \left\{\Upsilon_{2}, \Lambda_{2}, \Upsilon_{4}, \Lambda_{4}\right\}}\right)\right\} .
$$

Then

$$
\begin{aligned}
\|y\| \leq & \max \left\{\Upsilon_{1}, \Lambda_{1}, \Upsilon_{3}, \Lambda_{3}\right\} \\
& +\max \left\{\Upsilon_{2}, \Lambda_{2}, \Upsilon_{4}, \Lambda_{4}\right\} \Phi \\
& \times\left(\max \left\{W, \Phi^{-1}\left(\frac{\max \left\{\Upsilon_{1}, \Lambda_{1}, \Upsilon_{3}, \Lambda_{3}\right\}}{\max \left\{\Upsilon_{2}, \Lambda_{2}, \Upsilon_{4}, \Lambda_{4}\right\}}\right)\right\}\right) .
\end{aligned}
$$

It follows that $\Omega_{1}$ is bounded.

Case 2. Consider $\left(\left(\max \left\{\Upsilon_{2}, \Lambda_{2}, \Upsilon_{4}, \Lambda_{4}\right\} / w\left(\left(2 \max \left\{\Theta_{2}, \Sigma_{2}\right.\right.\right.\right.\right.$, $\left.\left.\left.\left.\Theta_{4}, \Sigma_{4}\right\}\right)^{-1}\right)\right)<1$ ).

Without loss of generality, suppose that

$$
\|y\| \geq \Phi\left(\frac{\max \left\{\Theta_{1}, \Sigma_{1}, \Theta_{3}, \Sigma_{3}\right\}}{\max \left\{\Theta_{2}, \Sigma_{2}, \Theta_{4}, \Sigma_{4}\right\}}\right) .
$$

Then using Remark 12 and the previous inequalities, we get

$$
\begin{aligned}
\|y\| \leq & \max \left\{\Upsilon_{1}, \Lambda_{1}, \Upsilon_{3}, \Lambda_{3}\right\} \\
& +\frac{\max \left\{\Upsilon_{2}, \Lambda_{2}, \Upsilon_{4}, \Lambda_{4}\right\}}{w\left(\left(2 \max \left\{\Theta_{2}, \Sigma_{2}, \Theta_{4}, \Sigma_{4}\right\}\right)^{-1}\right)}\|y\| .
\end{aligned}
$$

It follows that there exists a constant $W>0$ such that $\|y\| \leq$ $W$. We get

$$
\|y\| \leq \max \left\{W, \Phi\left(\frac{\max \left\{\Theta_{1}, \Sigma_{1}, \Theta_{3}, \Sigma_{3}\right\}}{\max \left\{\Theta_{2}, \Sigma_{2}, \Theta_{4}, \Sigma_{4}\right\}}\right)\right\} .
$$

Then

$$
\begin{aligned}
\|x\| \leq & \max \left\{\Theta_{1}, \Sigma_{1}, \Theta_{3}, \Sigma_{3}\right\} \\
& +\max \left\{\Theta_{2}, \Sigma_{2}, \Theta_{4}, \Sigma_{4}\right\} \Phi^{-1} \\
& \times\left(\max \left\{W, \Phi\left(\frac{\max \left\{\Theta_{1}, \Sigma_{1}, \Theta_{3}, \Sigma_{3}\right\}}{\max \left\{\Theta_{2}, \Sigma_{2}, \Theta_{4}, \Sigma_{4}\right\}}\right)\right\}\right) .
\end{aligned}
$$

It follows that $\Omega_{1}$ is bounded.

To apply Lemma 5 , let $\Omega$ be a nonempty open bounded subset of $X$ such that $\Omega \supset \overline{\Omega_{1}}$ centered at zero.

It is easy to see from Lemma 8 that $T$ is a completely continuous operator. One can see that

$$
(x, y) \neq \lambda T(x, y) \quad \forall(x, y) \in \partial \Omega, \lambda \in(0,1) .
$$

Thus, from Lemma $5,(x, y)=T(x, y)$ has at least one solution $(x, y) \in \bar{\Omega}$. So $(x, y)$ is a pair of solutions of BVP (3) and BVP (6). The proof of Theorem 14 is complete.

\section{Two Examples}

To illustrate the usefulness of our main result, we present two examples that Theorem 14 can readily apply.

Example 15. Consider the following impulsive boundary value problem:

$$
\begin{gathered}
D_{0^{+}}^{8 / 5} x(t)=t^{-1 / 5}(1-t)^{-1} \\
\quad \times\left(c+b t^{6 / 5}[y(t)]^{3}+a t^{9 / 5}\left[D_{0^{+}}^{1 / 5} y(t)\right]^{3}\right), \\
t \in(0,1), \quad t \neq \frac{1}{2}, \\
D_{0^{+}}^{9 / 5} y(t) \\
=t^{-1 / 5}(1-t)^{-1} \\
\times\left(c_{0}+b_{0} t^{1 / 15}[x(t)]^{1 / 3}+a_{0} t^{2 / 15}\left[D_{0^{+}}^{1 / 5} x(t)\right]^{1 / 3}\right), \\
\quad t \in(0,1), \quad t \neq t_{1}, \\
\lim _{t \rightarrow 0} t^{2 / 5} x(t)=G, \quad \lim _{t \rightarrow 0} t^{1 / 5} y(t)=H, \\
x(1)=M, \quad y(1)=N, \\
\Delta x\left(\frac{1}{2}\right)=c_{I}, \quad \Delta y\left(\frac{1}{2}\right)=c_{J}, \\
\Delta D_{0^{+}}^{1} x\left(\frac{1}{2}\right)=c_{1, I}, \quad \Delta D_{0^{+}}^{1} y\left(\frac{1}{2}\right)=c_{1, J},
\end{gathered}
$$

where $c, b, a, c_{0}, b_{0}, a_{0}, G_{0}, H_{0}, M_{0}, N_{0}, C_{I}, C_{J}, C_{1, I}, C_{1, J}$ are constants.

Corresponding to BVP (1), we have

(a) $\alpha=8 / 5, \beta=9 / 5, p=q=1 / 5$,

(b) $\phi(t)=\psi(t)=t^{-1 / 5}(1-t)^{-1 / 5}, f(t, U, V)=c+b t^{6 / 5} U^{3}+$ $a t^{9 / 5} V^{3}$ and $g(t, U, V)=c_{0}+b_{0} t^{1 / 15} U^{1 / 3}+a_{0} t^{2 / 15} V^{1 / 3}$ defined on $(0,1) \times R^{2}$, 
(c) $u(t)=v(t)=m(t)=n(t) \equiv 1, G(t, U, V)=G_{0}, H(t$, $U, V)=H_{0}, M(t, U, V)=M_{0}, N(t, U, V)=N_{0}$,

(d) $0=t_{0}<t_{1}=(1 / 2)<t_{2}=1$,

(e) $I(t, U, V)=c_{I}, I_{1}(t, U, V)=c_{1, I}, J(t, U, V)=c_{J}, J_{1}(t$, $U, V)=c_{1, J}$.

It is easy to show that

(A) $\phi$ satisfies $\alpha+2 \delta-q>0, \alpha+k+\delta-q \geq 0$, and $|\phi(t)| \leq L_{1} t^{k}(1-t)^{\delta}$ for all $t \in(0,1)$ with $L_{1}=1$ and $k=-(1 / 5)=\delta$;

$\psi$ satisfies $\eta+2 \theta-p>0, \beta+l+\theta-p \geq 0$, and $|\psi(t)| \leq L_{2} t^{l}(1-t)^{\theta}$ for all $t \in(0,1)$ with $L_{2}=1$ and $l=-(1 / 5)=\theta$;

(B) $f, G, M, I, I_{1}$ are $\beta$-Caratheodory functions and $g, H$, $N, J, J_{1}$ are $\alpha$-Caratheodory functions.

Furthermore, we have $\Phi^{-1}(x)=x^{3}$ and $\Phi(x)=x^{1 / 3}$ with $w(x)=x^{1 / 3}$ and $v(x)=x^{3}$. It is easy to see that

(i) the inequalities

$$
\begin{gathered}
\left|f\left(t, t^{\alpha-2} U, t^{\alpha-q-2} V\right)\right| \leq C_{f}+B_{f} \Phi^{-1}(|U|)+A_{f} \Phi^{-1}(|V|), \\
\left|G\left(t, t^{\alpha-2} U, t^{\alpha-q-2} V\right)\right| \leq C_{G}+B_{G} \Phi^{-1}(|U|)+A_{G} \Phi^{-1}(|V|), \\
\left|M\left(t, t^{\alpha-2} U, t^{\alpha-q-2} V\right)\right| \\
\leq C_{M}+B_{M} \Phi^{-1}(|U|)+A_{M} \Phi^{-1}\left(\left|\Phi^{-1}(|U|)\right|\right)
\end{gathered}
$$

hold for all $(U, V) \in R^{2}, t \in(0,1]$ with $C_{f}=|c|, B_{f}=$ $|b|, A_{f}=|a|, C_{G}=\left|G_{0}\right|, B_{G}=0, A_{G}=0$ and $C_{M}=$ $\left|M_{0}\right|, B_{M}=0, A_{M}=0$;

(ii) the inequalities

$$
\begin{gathered}
\left|g\left(t, t^{\beta-2} U, t^{\beta-p-2} V\right)\right| \leq C_{g}+B_{g} \Phi(U)+A_{g} \Phi(V), \\
\left|H\left(t, t^{\beta-2} U, t^{\beta-p-2} V\right)\right| \leq C_{H}+B_{H} \Phi(U)+A_{H} \Phi(V), \\
\left|N\left(t, t^{\beta-2} U, t^{\beta-p-2} V\right)\right| \leq C_{N}+B_{N} \Phi(U)+A_{N} \Phi(V)
\end{gathered}
$$

hold for all $(U, V) \in R^{2}, t \in(0,1]$ with $C_{g}=\left|c_{0}\right|$, $B_{g}=\left|b_{0}\right|, A_{g}=\left|a_{0}\right|, C_{H}=\left|H_{0}\right|, B_{H}=A_{H}=0$, $C_{N}=\left|N_{0}\right|, B_{N}=A_{N}=0$;

(iii) the inequalities

$$
\begin{gathered}
\left|I\left(t_{1}, t_{1}^{\alpha-2} U, t_{1}^{\alpha-q-2} V\right)\right| \leq C_{I}+B_{I} \Phi^{-1}(|U|)+A_{I} \Phi^{-1}(|V|), \\
\left|I_{1}\left(t_{1}, t_{1}^{\alpha-2} U, t_{1}^{\alpha-q-2} V\right)\right| \\
\leq C_{1, I}+B_{1, I} \Phi^{-1}(|U|)+A_{1, I} \Phi^{-1}(|V|)
\end{gathered}
$$

hold for all $(U, V) \in R^{2}$ with $C_{I}=\left|c_{I}\right|, B_{I}=A_{I}=0$, $C_{1, I}=\left|c_{1, I}\right|, B_{1, I}=A_{1, I}=0$; (iv) the inequalities

$$
\begin{gathered}
\left|J\left(t_{1}, t_{1}^{\beta-2} U, t_{1}^{\beta-p-2} V\right)\right| \leq C_{J}+B_{J} \Phi(U)+A_{J} \Phi(V), \\
\left|J_{1}\left(t_{1}, t_{1}^{\beta-2} U, t_{1}^{\beta-p-2} V\right)\right| \leq C_{1, J}+B_{1, J} \Phi(U)+A_{1, J} \Phi(V)
\end{gathered}
$$

hold for all $(U, V) \in R^{2}$ with $C_{J}=\left|c_{J}\right|, B_{J}=A_{J}=0$, $C_{1, J}=\left|c_{1, J}\right|, B_{1, J}=A_{1, J}=0$.

By direct computation, we know that

$$
\begin{aligned}
& \Theta_{2}=2 \frac{\mathbf{B}(7 / 5,4 / 5)}{\Gamma(8 / 5)}[|b|+|a|], \\
& \Sigma_{2}=2 \frac{\mathbf{B}(7 / 5,4 / 5)}{\Gamma(8 / 5)}[|b|+|a|],
\end{aligned}
$$$$
\Theta_{4}=\left(\frac{\mathbf{B}(6 / 5,4 / 5)}{\Gamma(7 / 5)}+\frac{\mathbf{B}(7 / 5,4 / 5)}{\Gamma(7 / 5)}\right)[|b|+|a|],
$$$$
\Sigma_{4}=\left(\frac{\mathbf{B}(6 / 5,4 / 5)}{\Gamma(7 / 5)}+\frac{\mathbf{B}(7 / 5,4 / 5)}{\Gamma(7 / 5)}\right)[|b|+|a|],
$$$$
\Upsilon_{2}=2 \frac{\mathbf{B}(8 / 5,4 / 5)}{\Gamma(9 / 5)}\left[\left|b_{0}\right|+\left|a_{0}\right|\right],
$$

$$
\Upsilon_{4}=\left(\frac{\mathbf{B}(7 / 5,4 / 5)}{\Gamma(8 / 5)}+\frac{\mathbf{B}(8 / 5,4 / 5)}{\Gamma(8 / 5)}\right)\left[\left|b_{0}\right|+\left|a_{0}\right|\right]
$$

$$
\Lambda_{2}=2 \frac{\mathbf{B}(8 / 5,4 / 5)}{\Gamma(9 / 5)}\left[\left|b_{0}\right|+\left|a_{0}\right|\right],
$$

$$
\Lambda_{4}=\left(\frac{\mathbf{B}(7 / 5,4 / 5)}{\Gamma(8 / 5)}+\frac{\mathbf{B}(8 / 5,4 / 5)}{\Gamma(8 / 5)}\right)\left[\left|b_{0}\right|+\left|a_{0}\right|\right] .
$$

Then Theorem 14 implies that the existence of at least one solution if

$$
\begin{aligned}
& \max \left\{2 \frac{\mathbf{B}(8 / 5,4 / 5)}{\Gamma(9 / 5)}, \frac{\mathbf{B}(7 / 5,4 / 5)}{\Gamma(8 / 5)}+\frac{\mathbf{B}(8 / 5,4 / 5)}{\Gamma(8 / 5)}\right\} \\
& \times\left(\max \left\{2 \frac{\mathbf{B}(7 / 5,4 / 5)}{\Gamma(8 / 5)}, \frac{\mathbf{B}(6 / 5,4 / 5)}{\Gamma(7 / 5)}+\frac{\mathbf{B}(7 / 5,4 / 5)}{\Gamma(7 / 5)}\right\}\right)^{1 / 3} \\
& \times\left[\left|b_{0}\right|+\left|a_{0}\right|\right][|b|+|a|]^{1 / 3}<\frac{1}{\sqrt[3]{2}} .
\end{aligned}
$$

Example 16. Consider the following boundary value problem without impulse effects:

$$
D_{0^{+}}^{7 / 4} x(t)=t^{-1 / 4}(1-t)^{-1 / 4}
$$

$$
\times\left(C+B t^{3 / 4}[y(t)]^{3}+A t^{15 / 4}\left[D_{0^{+}}^{1} y(t)\right]^{3}\right),
$$

$t \in(0,1)$ 


$$
\begin{aligned}
& D_{0^{+}}^{5 / 4} y(t) \\
& =t^{-1 / 8}(1-t)^{-1 / 8} \\
& \quad \times\left(C_{0}+B_{0} t^{1 / 4}[x(t)]^{1 / 3}+A_{0} t^{7 / 12}\left[D_{0^{+}}^{1 / 4} x(t)\right]^{1 / 3}\right), \\
& t \in(0,1), \\
& \lim _{t \rightarrow 0} t^{1 / 4} x(t)=0, \quad \lim _{t \rightarrow 0} t^{3 / 4} y(t)=0, \\
& x(1)=0, \quad y(1)=0,
\end{aligned}
$$

where $C, B, A, C_{0}, B_{0}$, and $A_{0}$ are constants.

Corresponding to BVP (1), we have

(a) $\alpha=7 / 4, \beta=5 / 4, p=1$ and $q=1 / 4$,

(b) $\phi(t)=t^{-1 / 4}(1-t)^{-1 / 4}, \psi(t)=t^{-1 / 8}(1-t)^{-1 / 8}$, $f, g$ defined on $(0,1) \times R^{2}, f(t, U, V)=C+$ $B t^{1 / 12} U^{3}+A t^{5 / 12} V^{3}$ and $g(t, U, V)=C_{0}+B_{0} t^{1 / 4} U^{1 / 3}+$ $A_{0} t^{7 / 12} V^{1 / 3}$

(c) $m(t)=n(t)=u(t)=v(t) \equiv 0, G(t, U, V)=H(t, U$, $V)=M(t, U, V)=N(t, U, V) \equiv 0$,

(d) there exists no impulse point,

(e) $I(t, U, V)=I_{1}(t, U, V)=J(t, U, V)=J_{1}(t, U, V) \equiv 0$.

It is easy to show that

(A) $\phi$ satisfies $\alpha+2 \delta-q>0, \alpha+k+\delta-q>0,|\phi(t)| \leq$ $L_{1} t^{k}(1-t)^{\delta}$ for all $t \in(0,1)$ with $L_{1}=1, k=-(1 / 4)=$ $\delta$;

$\psi$ satisfies $\beta+2 \theta-p>0, \beta+l+\theta-p \geq 0$, and $|\psi(t)| \leq L_{2} t^{l}(1-t)^{\theta}$ for all $t \in(0,1)$ with $L_{2}=1$, $l=-(1 / 8)=\theta$

(B) $f, G, M, I, I_{1}$ are $\beta$-Caratheodory functions and $g, H$, $N, J, J_{1}$ are $\alpha$-Caratheodory functions.

Furthermore, $\Phi(x)=x^{1 / 3}$ and $\Phi^{-1}(x)=x^{3}$, we have $w(x)=$ $x^{1 / 3}$ and $\nu(x)=x^{3}$, and

(i) the inequalities

$$
\begin{gathered}
\left|f\left(t, t^{\alpha-2} U, t^{\alpha-q-2} V\right)\right| \leq C_{f}+B_{f} \Phi^{-1}(|U|)+A_{f} \Phi^{-1}(|V|) \\
\left|G\left(t, t^{\alpha-2} U, t^{\alpha-q-2} V\right)\right| \leq C_{G}+B_{G} \Phi^{-1}(|U|)+A_{G} \Phi^{-1}(|V|) \\
\left|M\left(t, t^{\alpha-2} U, t^{\alpha-q-2} V\right)\right| \\
\leq C_{M}+B_{M} \Phi^{-1}(|U|)+A_{M} \Phi^{-1}\left(\left|\Phi^{-1}(|U|)\right|\right)
\end{gathered}
$$

hold for all $(U, V) \in R^{2}, t \in(0,1)$ with $C_{G}=B_{G}=$ $A_{G}=C_{M}=B_{M}=A_{M}=0, C_{f}=|C|, B_{f}=|B|$ and $A_{f}=|A|$; (ii) the inequalities

$$
\begin{gathered}
\left|g\left(t, t^{\beta-2} U, t^{\beta-p-2} V\right)\right| \leq C_{g}+B_{g} \Phi(U)+A_{g} \Phi(V), \\
\left|H\left(t, t^{\beta-2} U, t^{\beta-p-2} V\right)\right| \leq C_{H}+B_{H} \Phi(U)+A_{H} \Phi(V), \\
\left|N\left(t, t^{\beta-2} U, t^{\beta-p-2} V\right)\right| \leq C_{N}+B_{N} \Phi(U)+A_{N} \Phi(V)
\end{gathered}
$$

hold for all $(U, V) \in R^{2}, t \in(0,1)$ with $C_{H}=B_{H}=$ $A_{H}=C_{N}=B_{N}=A_{N}=0, C_{g}=\left|C_{0}\right|, B_{g}=\left|B_{0}\right|$ and $A_{g}=\left|A_{0}\right|$;

(iii) the inequalities

$$
\begin{gathered}
\left|I\left(t_{1}, t_{1}^{\alpha-2} U, t_{1}^{\alpha-q-2} V\right)\right| \\
\leq C_{I}+B_{I} \Phi^{-1}(|U|)+A_{I} \Phi^{-1}(|V|), \\
\left|I_{1}\left(t_{1}, t_{1}^{\alpha-2} U, t_{1}^{\alpha-q-2} V\right)\right| \\
\quad \leq C_{1, I}+B_{1, I} \Phi^{-1}(|U|)+A_{1, I} \Phi^{-1}(|V|)
\end{gathered}
$$

hold for all $(U, V) \in R^{2}$ with $C_{I}=B_{I}=A_{I}=C_{1, I}=$ $B_{1, I}=A_{1, I}=0$;

(iv) there exist the nonnegative numbers $A_{i, k}, B_{i, k}$, $C_{i, k}(i=1,2)$ such that

$$
\left|J\left(t_{1}, t_{1}^{\beta-2} U, t_{1}^{\beta-p-2} V\right)\right| \leq C_{J}+B_{J} \Phi(U)+A_{J} \Phi(V),
$$

$\left|J_{1}\left(t_{1}, t_{1}^{\beta-2} U, t_{1}^{\beta-p-2} V\right)\right| \leq C_{1, J}+B_{1, J} \Phi(U)+A_{1, J} \Phi(V)$

hold for all $(U, V) \in R^{2}$ with $C_{J}=B_{J}=A_{J}=C_{1, J}=$ $B_{1, J}=A_{1, J}=0$.

By direct computation, we know that

$$
\begin{aligned}
& \Theta_{2}=2 \frac{\mathbf{B}(3 / 2,3 / 4)}{\Gamma(7 / 4)}[|B|+|A|], \\
& \Sigma_{2}=2 \frac{\mathbf{B}(3 / 2,3 / 4)}{\Gamma(7 / 4)}[|B|+|A|],
\end{aligned}
$$

$$
\Theta_{4}=\left(\frac{\mathbf{B}(5 / 4,3 / 4)}{\Gamma(3 / 2)}+\frac{\mathbf{B}(3 / 2,3 / 4)}{\Gamma(3 / 2)}\right)[|B|+|A|],
$$$$
\Sigma_{4}=\left(\frac{\mathbf{B}(5 / 4,3 / 4)}{\Gamma(3 / 2)}+\frac{\mathbf{B}(3 / 2,3 / 4)}{\Gamma(3 / 2)}\right)[|B|+|A|],
$$

$$
\Upsilon_{2}=2 \frac{\mathbf{B}(9 / 8,7 / 8)}{\Gamma(5 / 4)}\left[\left|B_{0}\right|+\left|A_{0}\right|\right]
$$

$\Upsilon_{4}=\left(\frac{\mathbf{B}(1 / 8,7 / 8)}{\Gamma(\beta-p)}+\frac{\mathbf{B}(9 / 8,7 / 8)}{\Gamma(1 / 4)}\right)\left[\left|B_{0}\right|+\left|A_{0}\right|\right]$,

$$
\Lambda_{2}=2 \frac{\mathbf{B}(9 / 8,7 / 8)}{\Gamma(5 / 4)}\left[\left|B_{0}\right|+\left|A_{0}\right|\right]
$$

$\Lambda_{4}=\left(\frac{\mathbf{B}(1 / 8,7 / 8)}{\Gamma(1 / 4)}+\frac{\mathbf{B}(9 / 8,7 / 8)}{\Gamma(1 / 4)}\right)\left[\left|B_{0}\right|+\left|A_{0}\right|\right]$. 
Then Theorem 14 implies the existence of at least one solution if

$$
\begin{aligned}
\max & \left\{2 \frac{\mathbf{B}(3 / 2,3 / 4)}{\Gamma(7 / 4)}, \frac{\mathbf{B}(5 / 4,3 / 4)}{\Gamma(3 / 2)}+\frac{\mathbf{B}(3 / 2,3 / 4)}{\Gamma(3 / 2)}\right\} \\
& \times\left(\max \left\{2 \frac{\mathbf{B}(9 / 8,7 / 8)}{\Gamma(5 / 4)}, \frac{\mathbf{B}(1 / 8,7 / 8)}{\Gamma(\beta-p)}+\frac{\mathbf{B}(9 / 8,7 / 8)}{\Gamma(1 / 4)}\right\}\right)^{3} \\
& \times\left[\left|B_{0}\right|+\left|A_{0}\right|\right]^{3}[|B|+|A|]<\frac{1}{8} .
\end{aligned}
$$

Remark 17. It is easy to see that the previous boundary value problems have at least one solution for sufficiently small $\left|B_{1}\right|,\left|B_{2}\right|$ and $\left|A_{0}\right|,\left|B_{0}\right|,|a|,|b|,\left|a_{0}\right|$ and $\left|b_{0}\right|$. They cannot be solved by the theorems in $[24,25]$.

\section{Acknowledgments}

This research is partially supported by the Natural Science Foundation of Guangdong province (no. S2011010001900) and the Guangdong Higher Education Foundation for HighLevel Talents. This research is partially supported by Ministerio de Economía y Competitividad and EC fund FEDER, Project no. MTM2010-15314, Spain.

\section{References}

[1] K. S. Miller and B. Ross, An Introduction to the Fractional Calculus and Fractional Differential Equations, Wiley, New York, NY, USA, 1993.

[2] S. G. Samko, A. A. Kilbas, and O. I. Marichev, Fractional Integral and Derivative. Theory and Applications, Gordon and Breach, 1993.

[3] A. A. Kilbas, H. M. Srivastava, and J. J. Trujillo, Theory and Applications of Fractional Differential Equations, vol. 204 of North-Holland Mathematics Studies, Elsevier Science B.V., Amsterdam, The Netherlands, 2006.

[4] B. Ahmad and J. J. Nieto, "Anti-periodic fractional boundary value problems with nonlinear term depending on lower order derivative," Fractional Calculus and Applied Analysis, vol. 15, no. 3, pp. 451-462, 2012.

[5] B. Ahmad, J. J. Nieto, A. Alsaedi, and M. El-Shahed, "A study of nonlinear Langevin equation involving two fractional orders in different intervals," Nonlinear Analysis: Real World Applications, vol. 13, no. 2, pp. 599-606, 2012.

[6] I. Podlubny and N. Heymans, "Physical interpretation of initial conditions for fractional differential equations with RiemannLiouville fractional derivatives," Rheologica Acta, vol. 45, no. 5, pp. 765-771, 2006.

[7] I. Podlubny, "Geometric and physical interpretation of fractional integration and fractional differentiation," Fractional Calculus and Applied Analysis, vol. 5, no. 4, pp. 367-386, 2002.

[8] A. Carpinteri and F. Mainardi, Eds., Fractals and Fractional Calculus in Continuum Mechanics, CISM Courses and Lectures, International Center for Mechanical Sciences, no. 378, Springer, New York, NY, USA, 1997.
[9] R. L. Magin, "Fractional calculus in bioengineering, part 1," Critical Reviews in Biomedical Engineering, vol. 32, no. 1, pp. 1104, 2004.

[10] R. L. Magin, "Fractional calculus in bioengineering, part 2," Critical Reviews in Biomedical Engineering, vol. 32, no. 1, pp. 105193, 2004.

[11] R. L. Magin, "Fractional calculus in bioengineering, part 3," Critical Reviews in Biomedical Engineering, vol. 32, no. 3-4, pp. 195-377, 2004.

[12] X. Chen, L. Wei, J. Sui, and L. Zheng, "Solving the linear timefractional wave equation by generalized differential transform method," Applied Mechanics and Materials, vol. 204-208, pp. 4476-4480, 2012.

[13] S. Li, B. Fang, T. Yang, Y. Zhang, L. Tan, and W. Huang, "Dynamics of vibration isolation system obeying fractional differentiation," Aircraft Engineering and Aerospace Technology, vol. 84, no. 2, pp. 103-108, 2012.

[14] G. S. Priya, P. Prakash, J. J. Nieto, and Z. Kayar, "Higher order numerical scheme for fractional heat equation with Dirichlet and Neumann boundary conditions," Numerical Heat Transfer $B$. In press.

[15] J. Zhao, B. Tang, S. Kumar, and Y. Hou, "The extended fractional subequation method for nonlinear fractional differential equations," Mathematical Problems in Engineering, vol. 2012, Article ID 924956, 11 pages, 2012.

[16] V. V. Lakshmikantham, D. D. Baĭnov, and P. S. Simeonov, Theory of Impulsive Differential Equations, World Scientific, Singapore, 1989.

[17] V. Kavitha and M. Mallika Arjunan, "Controllability of impulsive quasi-linear fractional mixed Volterra-Fredholm-type integrodifferential equations in Banach spaces," The Journal of Nonlinear Science and Its Applications, vol. 4, no. 2, pp. 152-169, 2011.

[18] B. Ahmad and S. Sivasundaram, "Existence results for nonlinear impulsive hybrid boundary value problems involving fractional differential equations," Nonlinear Analysis: Hybrid Systems, vol. 3, no. 3, pp. 251-258, 2009.

[19] B. Ahmad and S. Sivasundaram, "Existence of solutions for impulsive integral boundary value problems of fractional order," Nonlinear Analysis: Hybrid Systems, vol. 4, no. 1, pp. 134$141,2010$.

[20] B. Ahmad and J. J. Nieto, "Existence of solutions for impulsive anti-periodic boundary value problems of fractional order," Taiwanese Journal of Mathematics, vol. 15, no. 3, pp. 981-993, 2011.

[21] Y. Tian and Z. Bai, "Existence results for the three-point impulsive boundary value problem involving fractional differential equations," Computers \& Mathematics with Applications, vol. 59, no. 8, pp. 2601-2609, 2010.

[22] R. P. Agarwal, M. Benchohra, and S. Hamani, "A survey on existence results for boundary value problems of nonlinear fractional differential equations and inclusions," Acta Applicandae Mathematicae, vol. 109, no. 3, pp. 973-1033, 2010.

[23] J. Mawhin, Topological Degree Methods in Nonlinear Boundary Value Problems, vol. 40 of NSFCBMS Regional Conference Series in Mathematics, American Mathematical Society, Providence, RI, USA, 1979.

[24] B. Ahmad and J. J. Nieto, "Existence results for a coupled system of nonlinear fractional differential equations with threepoint boundary conditions," Computers \& Mathematics with Applications, vol. 58, no. 9, pp. 1838-1843, 2009. 
[25] X. Su, "Boundary value problem for a coupled system of nonlinear fractional differential equations," Applied Mathematics Letters, vol. 22, no. 1, pp. 64-69, 2009.

[26] G. L. Karakostas, "Positive solutions for the $\Phi$-Laplacian when $\Phi$ is a sup-multiplicative-like function," Electronic Journal of Differential Equations, vol. 2004, no. 68, pp. 1-12, 2004. 


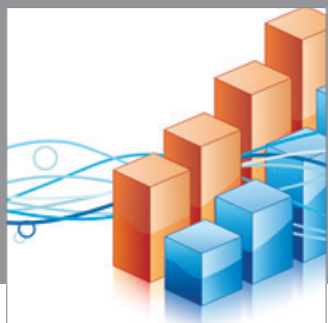

Advances in

Operations Research

mansans

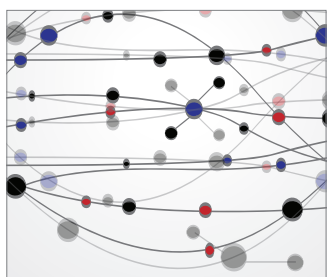

The Scientific World Journal
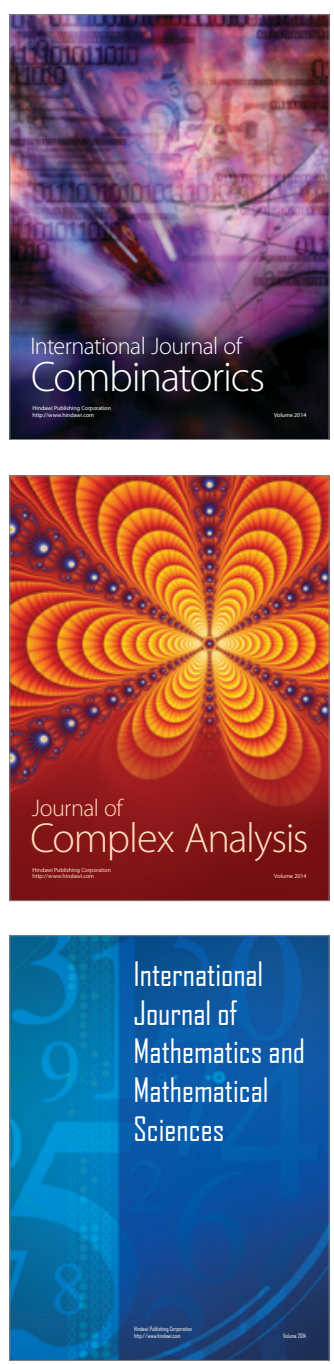
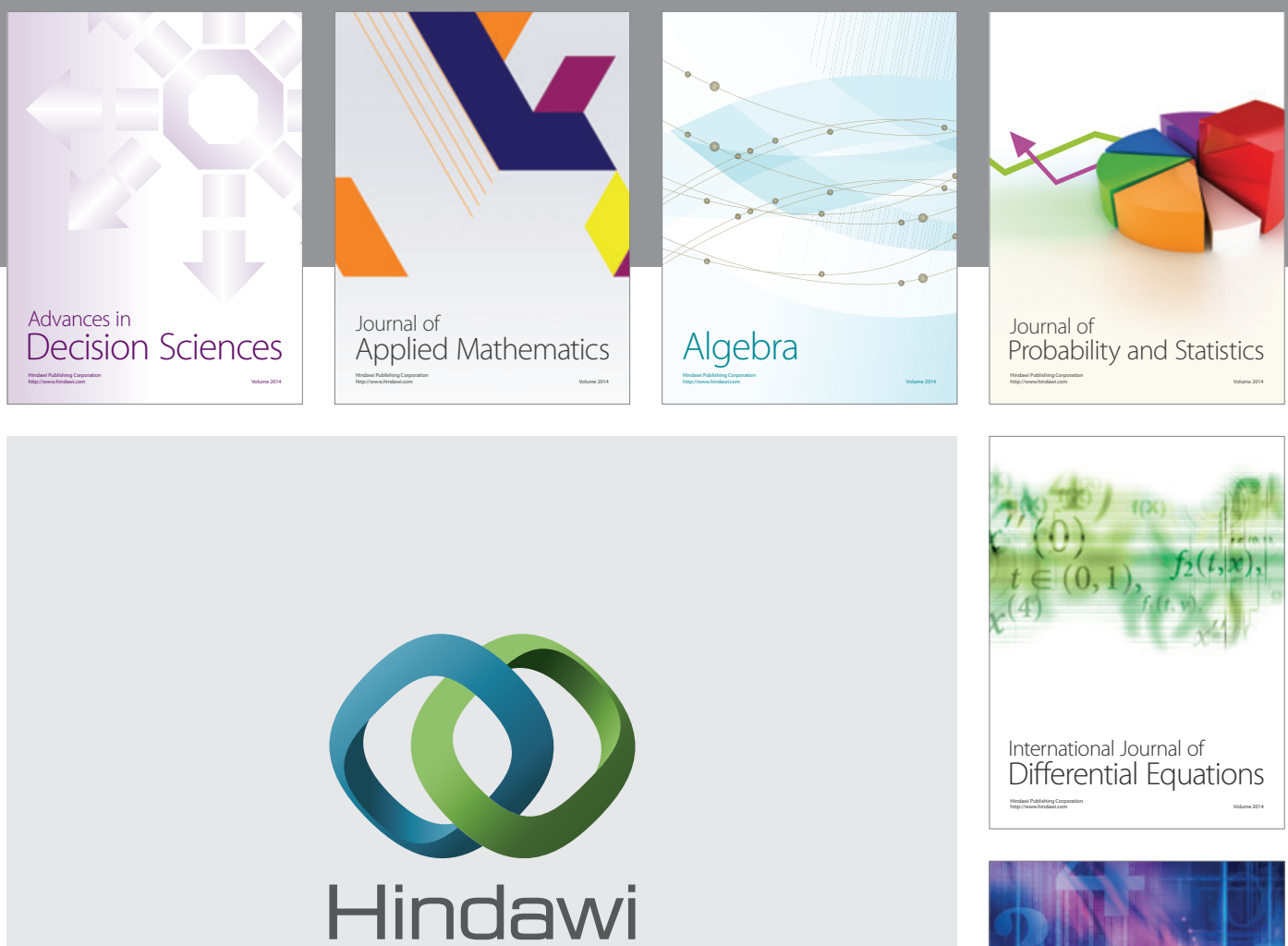

Submit your manuscripts at http://www.hindawi.com
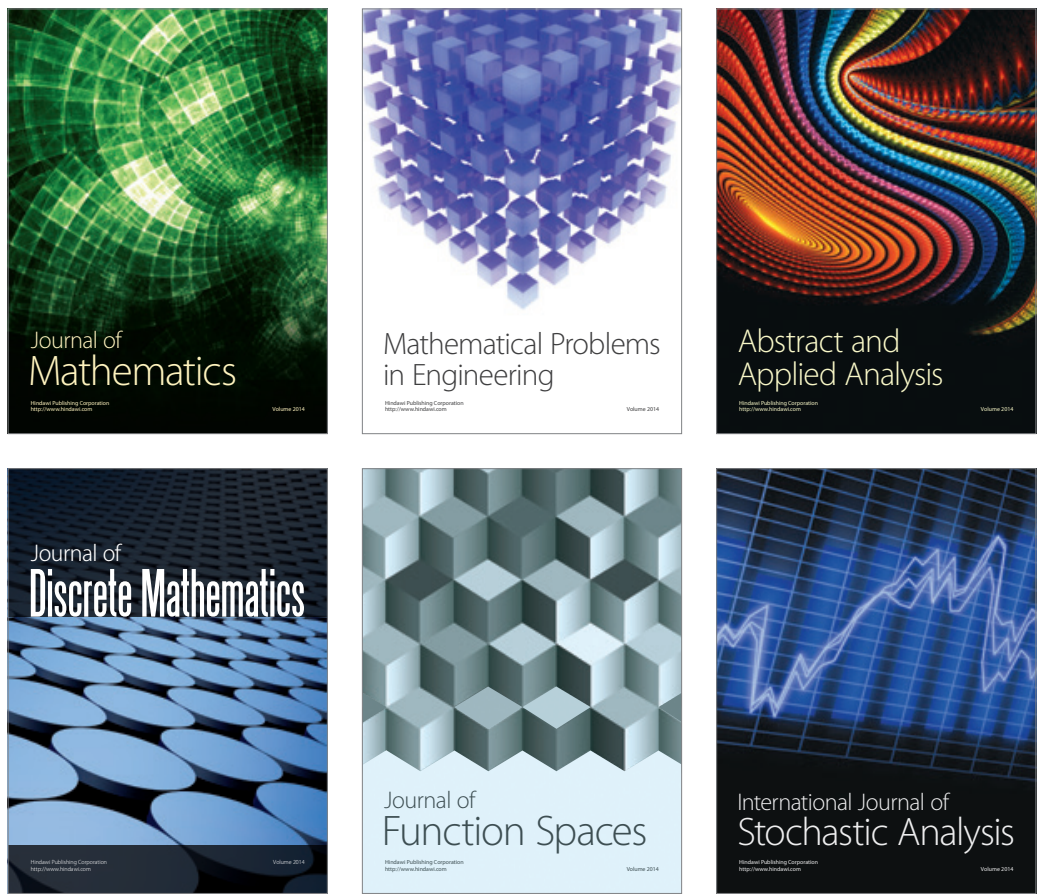

Journal of

Function Spaces

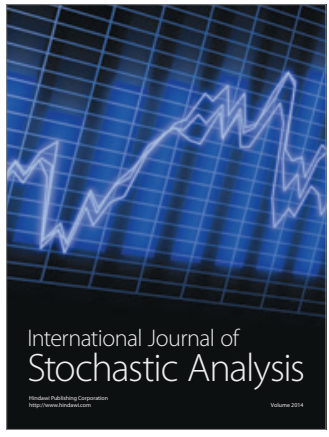

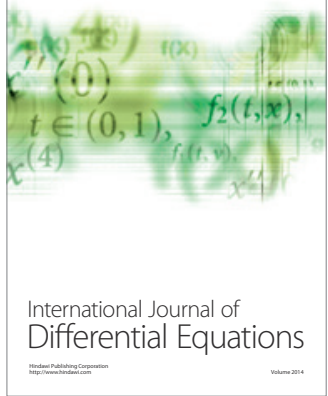
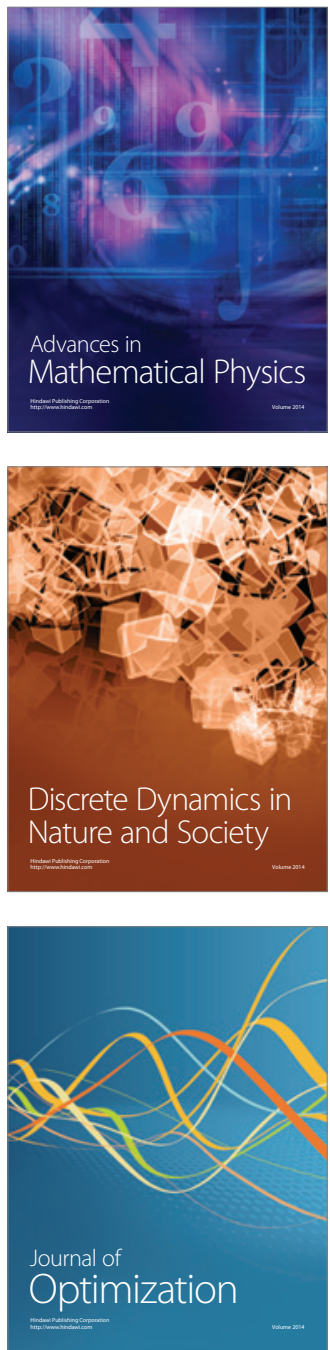